\title{
Learning 3D Semantic Segmentation with only 2D Image Supervision
}

\author{
Kyle Genova ${ }^{1,2}$ Xiaoqi Yin ${ }^{1}$ Abhijit Kundu ${ }^{1}$ Caroline Pantofaru ${ }^{1}$ Forrester Cole $^{1}$ \\ Avneesh Sud ${ }^{1}$ Brian Brewington ${ }^{1}$ Brian Shucker ${ }^{1}$ Thomas Funkhouser $^{1,2}$ \\ ${ }^{1}$ Google Research $\quad{ }^{2}$ Princeton University
}

\begin{abstract}
With the recent growth of urban mapping and autonomous driving efforts, there has been an explosion of raw $3 D$ data collected from terrestrial platforms with lidar scanners and color cameras. However, due to high labeling costs, ground-truth $3 D$ semantic segmentation annotations are limited in both quantity and geographic diversity, while also being difficult to transfer across sensors. In contrast, large image collections with ground-truth semantic segmentations are readily available for diverse sets of scenes. In this paper, we investigate how to use only those labeled $2 D$ image collections to supervise training $3 D$ semantic segmentation models. Our approach is to train a $3 D$ model from pseudo-labels derived from $2 D$ semantic image segmentations using multiview fusion. We address several novel issues with this approach, including how to select trusted pseudo-labels, how to sample $3 D$ scenes with rare object categories, and how to decouple input features from $2 D$ images from pseudo-labels during training. The proposed network architecture, 2D3DNet, achieves significantly better performance $(+6.2-11.4 \mathrm{mIoU})$ than baselines during experiments on a new urban dataset with lidar and images captured in 20 cities across 5 continents.
\end{abstract}

\section{Introduction}

Semantic segmentation of 3D scenes is a fundamental task in computer vision with applications in semantic mapping, logistics planning, augmented reality, and several other domains. In these applications, the input data is typically a sequence of laser scans and posed color images acquired from a terrestrial platform, and the task is to predict a category label for every $3 \mathrm{D}$ point (Figure 1 ). In this work, we aim for a method that generalizes to real-world data captured with any sensor anywhere in the world.

This task is challenging due in part to the difficulty of obtaining training datasets with dense 3D semantic anno-

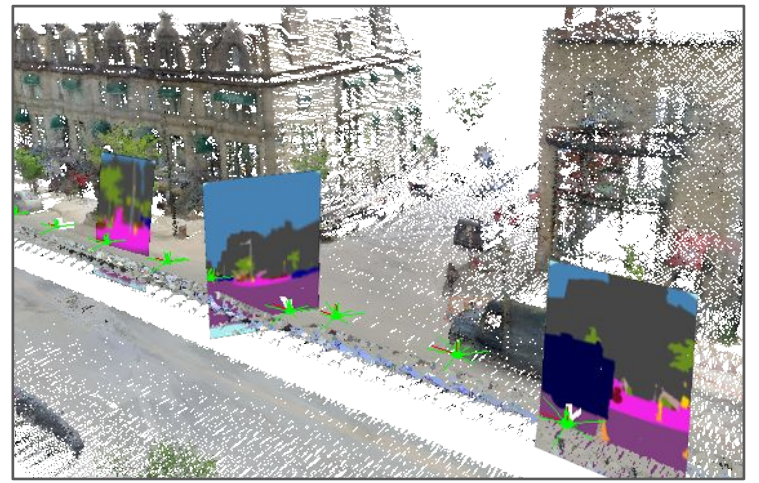

Input: unlabeled 3D + 2D predicted segmentation

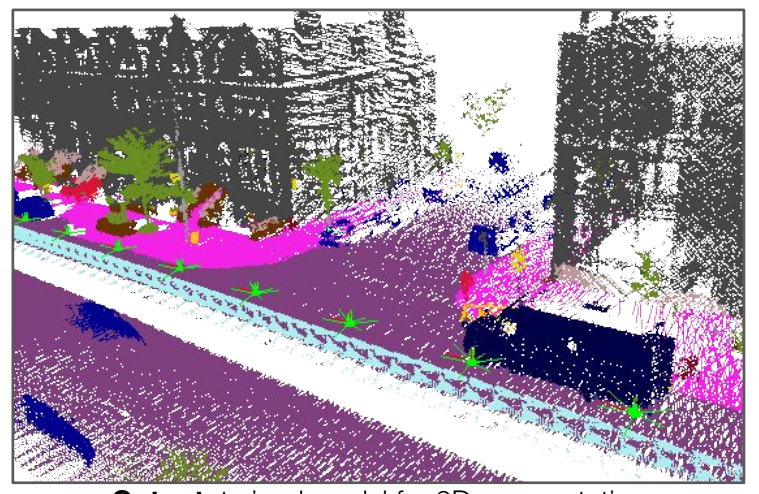

Output: trained model for 3D segmentation

Figure 1: Semantic segmentation of 3D data without any 3D supervision. We leverage semantic segmentations of posed 2D images (top) to produce pseudo-labels to train a model for semantic segmentation of $3 \mathrm{D}$ point clouds (bottom).

tations. Several 3D semantic segmentation benchmarks have been released for outdoor scenes in the past few years $[7,10,31,67,37,73]$. However, they each contain data captured with a unique lidar sensor configuration in a unique part of the world. As a result, training on one of these datasets and then inferring on another performs poorly, as models fit to the specific sampling patterns and unique content of their training data (see Appendix C). While one could try unsupervised domain adaptation, generalization to 
new lidar sensor configurations is notoriously difficult, and SOTA domain adaptation methods are not competitive for semantic segmentation of 3D point clouds [92].

In this work, we aim for a 3D semantic segmentation method that works in-the-wild for data captured with any lidar sensor anywhere in the world, even where no 3D semantic annotations are available. This focus is motivated by the needs of world mapping organizations, which continuously collect massive stores of unlabeled 3D lidar and image data from around the globe with a variety of sensors, but do not have ground-truth 3D annotations for it.

Our solution is to leverage labeled 2D image collections. There are a number of 2D image datasets with per-pixel semantic labels [19, 55], some of which are quite large and diverse, with images from around the world [64]. Supervised 2D models trained on these datasets have been shown to generalize well to images from diverse settings [53]. Our approach is to use these trained 2D models to produce pseudo-labels for training 3D models. In this way, we can train a 3D semantic segmentation model uniquely for each $3 \mathrm{D}$ dataset without requiring any $3 \mathrm{D}$ annotations.

Our network architecture, which we call 2D3DNet, is shown in Figure 2. For each 3D scene in an unlabeled training set, we first run a pre-trained 2D model on every image to produce a semantic label for each pixel. Then for each 3D point, we back-project it into every image to produce a set of weighted votes, which are tallied to produce features and also pseudo-labels. Finally, we train a 3D model that takes in 3D points with features and outputs semantic labels using supervision from the pseudo-labels. At test time, we run the 2D model to produce features (optionally), and then the 3D model to predict a final 3D semantic segmentation.

Although this basic method addresses the main issue (how to supervise 3D with 2D), it has several issues. First, pseudo-labels can be very noisy, partly due to errors made by $2 \mathrm{D}$ models, and partly due to errors in 2D-3D correspondences. It is not enough to simply distill a 3D model from corresponding 2D pixel predictions (see Section 5.1). Second, training a 3D network that takes in features from 2D images and predicts pseudolabels from the same images can create a correlation that impedes learning. Of course, features from $2 \mathrm{D}$ images are critical to performing $3 \mathrm{D}$ semantic segmentation, since many classes are easier to discriminate in images than point clouds (see Table 7 in the Appendix). Finally, because ground truth 3D annotations are not required, the potential training datasets are massive, raising interesting questions of how to sample training data.

We have addressed these issues by creating trusted sparse pseudo-labels, preventing feature coupling, and optimizing our dataset for rare and interesting examples. The result is a generalizable 3D model with supervision only from unpaired 2D images. We train the model on an unlabeled dataset of 250,000 scenes, and test it on a diverse set of scenes from 20 cities in five continents. This approach improves over baselines by $6.2-11.4 \mathrm{mIoU}$, and is competitive with state of the art methods on the nuScenes lidarseg benchmark while reducing its reliance on 3D annotations.

\section{Related Work}

3D semantic segmentation: Many researchers have worked on 3D semantic segmentation [11, 29, 56, 20, 62, 95]. For outdoor scenes, most early work focused on separately labeling terrain $[58,82,88]$, roads $[18,34,41,93,97]$, buildings $[8,12,24,51,65]$, trees $[87,91]$, and standalone objects [27]. Recent work has focused on 3D network architectures to extract features from 3D point clouds [86, 69, 70, 71, 76, 80, 38], meshes [33, 39], voxels [78], octrees [72], and sparse grids [16, 17, 28, 32]. Yet, all of these methods require expensive labeled 3D data for training.

3D semantic segmentation datasets. Some datasets have been released with ground-truth 3D semantic segmentation labels in the past few years. Examples include Paris-Lille3D [73] (2km in two cities in France), SemanticKITTI [7] (40km near Karlsruhe), Pandaset [37] (a few sequences near San Francisco), and NuScenes Lidarseg [10] (1000 scenes from one neighborhood in Boston and three in Singapore). These datasets lack diversity, with most covering one or two small regions. Plus, they are all captured with different lidar configurations, which makes cross-dataset training and domain adaptation difficult [1, 3, 44, 43, 90]. In contrast, labeled 2D datasets have great diversity. For example, Mapillary Vistas has labeled images from 25,000 scenes across six continents [64]. We leverage the size and diversity of image datasets to train generalizable 3D models.

Multi-view fusion. Previous approaches have explored aggregating $2 \mathrm{D}$ image semantic features onto $3 \mathrm{D}$ points using weighted averaging [36, 50, 84, 5], CRFs [60], label diffusion [59], and Bayesian fusion [57, 84, 94]. However, these previous works do not extract any features in the 3D domain, and thus they under-perform at recognizing objects with distinctive 3D shapes. Plus, they do not produce a trained 3D model that can be applied to novel scenes without posed images.

Hybrid 2D-3D convolutions. Some existing methods include 3D convolutions after multi-view fusion of 2D image features $[42,22,52,85]$. These methods follow the same basic approach as ours. However, they do not address the issues at the heart of our work. They require full 3D supervision, they assume good correspondence between 2D and $3 \mathrm{D}$, and they consider only datasets with small scenes [52], indoor settings [22, 42], or foreground objects [85]. These methods would not generalize or scale to outdoor scenes 


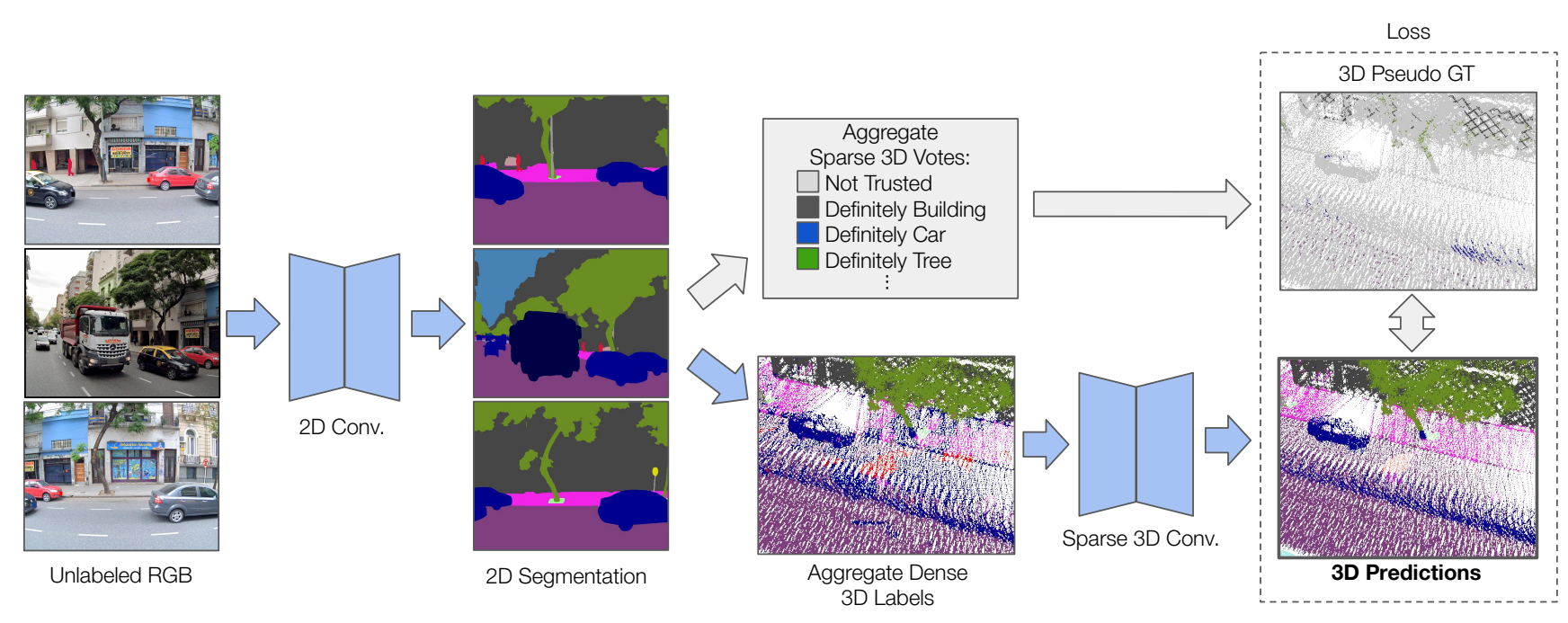

Figure 2: Our approach is composed of three stages. First, we run a pretrained 2D segmentation network on the input RGB images. Second, we aggregate the images into both dense, noisy segmentations and sparse, clean segmentations. Third, the dense segmentations are passed to a 3D sparse voxel convolution network, and a standard softmax cross-entropy loss is applied at the sparse locations.

from anywhere in the world.

Cross-modal supervision. This work sits within the area of cross-modal supervision, using information in one modality to train models in another modality through correspondence. Another example of this approach is in the video domain, where temporal synchronization provides correspondences between audio and visual, e.g. [66, 96, 49, 25, 6, 23, 47, 4] or text, speech and visual signals, e.g. [63]. There are also methods that transfer supervision or share information from image models to other domains- depth and RGB [30, 61], image and video [26], or image and 3D [81, 45, 54]. However, these image-based transfer approaches rely on the assumption that accurate correspondence is known (e.g., paired images). Here, the correspondence between modalities is only a sparse, noisy estimate.

\section{Methods}

Our network architecture, 2D3DNet, is composed of three stages (Figure 2). It takes as input a 2D semantic segmentation model ("2D Conv.") pretrained on a labeled image collection and a "scene" containing a short $\sim 10$ s sequence of unlabeled RGB images and lidar points captured contemporaneously, but asynchronously (Figure 1). In the first stage, it uses the pretrained 2D model to create 2D segmentation predictions for all image pixels. In the second stage, it uses multi-view fusion to make best-guess semantic labels for as many $3 \mathrm{D}$ points as possible via backprojection and voting from labels of the corresponding pixels. In the third stage, those aggregated dense 3D labels are converted to input features at the $3 \mathrm{D}$ points and run through a sparse 3D convolutional network (see Appendix G.2 for architecture) to predict the semantic label for every $3 \mathrm{D}$ point.

Given this inference model, the main challenge is how to train it. Previous models of this type are trained with supervision from in-domain labeled 3D data. However, most real-world scenarios will not have such data. Instead, they have large repositories of unlabeled data. To use unlabeled data to train the 3D model, we generate 3D pseudo-groundtruth training data from predicted 2D semantic segmentations (Section 3.1), carefully decouple the 2D segmentation image input features from the 3D pseudo-labels to ensure the 3D model generalizes beyond the training data (Section 3.2), and select unlabeled scenes judiciously so that the trained model performs well on rare semantic categories (Section 3.3). Remaining implementation details are provided in Appendix G.

\subsection{Generating 3D Pseudo-Ground-Truth}

Our first problem is to create pseudo-ground-truth labels for 3D points in the training set. Given a scene with predicted 2D semantic labels for all pixels of all images, our goal is to produce labels for 3D points that can be used to train a 3D semantic segmentation model.

Although it seems simple to backproject the predicted labels for all 2D pixels onto 3D points and vote, this naïve approach does not work well for several reasons (see results in Section 5.1). First, 3D lidar sweeps $(10 \mathrm{~Hz})$ and $2 \mathrm{D}$ images $(2 \mathrm{~Hz})$ are captured asynchronously with moving sensors, and thus there is no obvious one-to-one correspondence between 3D points and 2D pixels. Second, realworld urban scenes contain dynamic objects that can move significantly between 2D image and 3D lidar observations (cars, people, etc.), and thus observations at time A by one sensor may be inconsistent with observations at time B by the other (Figure 3a). Third, predicted semantic labels in 2D images are almost never pixel-perfect, with labels that bleed across object boundaries and lead to incorrect back- 
projections near occlusion boundaries (Figure $3 b$ ). Fourth, ground truth standards between 2D and 3D dataset annotation differ, for example fences are mostly see-through but usually segmented as solid in 2D for practical reasons (see Figure 3c), leading to inaccurate backprojection to 3D.

Our solution is to create pseudo-ground-truth for only the extremely sparse set of 3D points for which 2D-3D correspondences can be established robustly. The key observation is that unlabeled scene data is abundant, so we can throw out labels for $99 \%$ or more of the 3D points and still have more than enough data to train a 3D model. The goal then is to find the point-pixel correspondences we should trust. To implement this idea, we use a series of filters to exclude questionable point-pixel correspondences.

Temporal Filter. First, we exclude points whose timestamps are more than $\Delta t_{\max }$ from that of the closest image (by default $\Delta t_{\max }=0.1$ seconds). Since images are captured sparsely in time ( $2 \mathrm{~Hz}$ in our data), this filter removes the majority of the lidar points. It also removes the most egregious types of correspondence errors caused by moving objects. For example, in Figure 3a, points on the road behind the moving bus observed before or after the bus drove by will not be matched with the "bus" pixels in the image.

Occlusion Filter. Second, we apply an occlusion test that conservatively over-estimates the extent of foreground objects. To check for occlusion, we render a depth channel per image where the lidar points are drawn as discs (surfels [68]) with normals and two radii expressing the size and aspect ratio. The normals and radii are estimated per surfel by computing the size and aspect ratio of the local neighborhood of points using PCA (see Appendix G.3 for details). After rendering depth images, a point is considered occluded if its projected depth mismatches the depth image by a factor exceeding $\tau=1 \%$. To conservatively overestimate the extent of foreground objects, we purposely multiply ("dilate") the base estimated radii of the lidar discs by a factor of $k=8$. A conservative test is needed for cases such as the red points in Figure 3b,c, which would otherwise receive incorrect predicted class labels because of imperfect segmentation masks.

Weighted Voting. Third, we aggregate the $2 \mathrm{D}$ predicted labels that passed the filters to determine the pseudo-groundtruth 3D point labels. The scheme up-weights near-in-time, near-to-camera views while still including more spatially or temporally distant observations if they are all that is available. Concretely, given thresholds for the maximum distance $d_{\max }$ and maximum timestamp difference $\Delta t_{\max }$, the weight for an image- $i$, point $-j$ pair is:

$$
w_{i j}=\left(1-\frac{\left\|p_{i}^{c a m}-p_{j}^{p t}\right\|_{2}^{2}}{d_{\max }^{2}}\right)^{2}\left(1-\frac{\left|t_{i}^{c a m}-t_{j}^{p t}\right|^{2}}{\Delta t_{\max }^{2}}\right)^{2}
$$

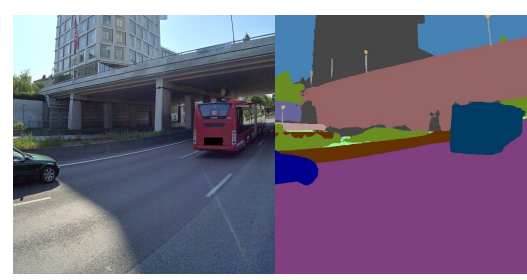

a1) RGB

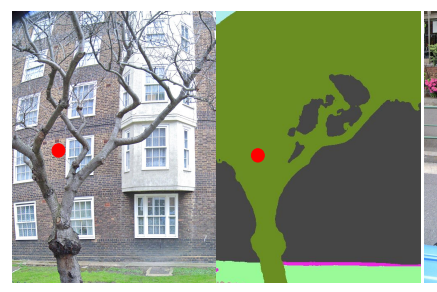

b1) RGB b2) Prediction

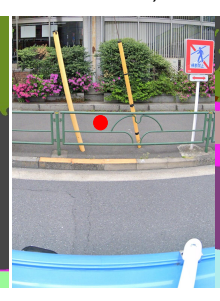

c1) RGB

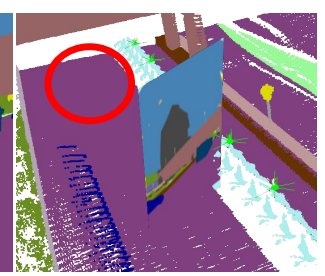

a3) Posed Image + GT Lidar
Figure 3: Challenges of creating 3D supervision from 2D votes. (a) A car and a bus drive forward with the ego vehicle. Because of aliasing, the car has an extended lidar point cloud, while the bus is completely absent from the lidar (red circle). (b) The network frequently makes mistakes near occlusion boundaries, so points that are near the silhouette of an occluder are not trusted. (c) Labeling standards differ from 2D to 3D. In 2D, fences are typically filled completely, regardless of their density.

In combination, these filters exclude almost all 3D points from the pseudo-ground-truth set, as designed. By applying the filters to vast amounts of unlabeled data, we produce a very large training set with reliable pseudo-ground-truth. Our filters are very conservative and thus produce few false positives that could affect the 3D network training process.

\subsection{Decoupling Image-Based Input Features from Image-Based Pseudo-Ground-Truth Labels}

The second main issue is how best to use features derived from RGB images as input to the 3D network. Features extracted from RGB images are very useful for 3D semantic scene understanding since many object classes are more easily recognized by their appearance in color images than by their geometric shapes (e.g., crosswalks). We demonstrate this quantitatively in the Appendix $(+28.9 \mathrm{mIoU}$ for our semantic 2D features, Table 7).

It would be natural to backproject semantic segmentation features extracted from 2D images, fuse them on 3D points and provide them as input channels for the $3 \mathrm{D}$ model. But if this process matches the process used to create the pseudoground-truth labels, the input features and supervision will always agree at sparse supervision locations. A network trained with these input features can minimize its loss by simply passing through the input features without doing any geometric reasoning. Such a pass-through function is not useful outside the sparse pseudo-ground truth set. ${ }^{1}$

\footnotetext{
${ }^{1}$ Note that this problem is not encountered when 3D labels are available. It occurs when the same set of image-based semantic segmentations are used to create both the input features and the ground truth.
} 


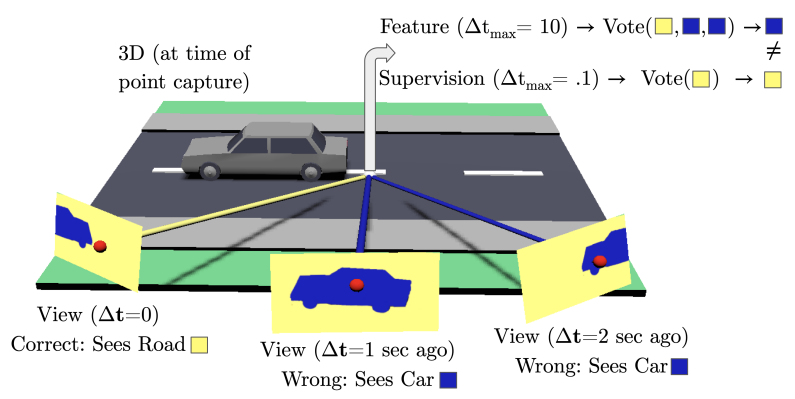

Figure 4: Illustration of how input features and pseudo-ground truth are generated for a point on the road. Input features are created by voting with multiview fusion using all images - in this case 2 out of 3 images vote "car." Pseudo-ground truth is created by voting only with images captured at nearly the same time (small $\Delta \mathrm{t}$ ) and with a clear line of sight (not near silhouettes) - in this case, only the left image qualifies and the result is "road." The $3 \mathrm{D}$ network is trained to predict the pseudo-ground truth from the input features - i.e., fix the mistakes of multi-view fusion.

To address this issue, we aggregate input features for the network and pseudo-ground-truth labels using two independent multiview fusion steps that have different parameters. Specifically, when doing fusion for features, we increase the max timestamp difference $\Delta t_{\max }$ substantially from 0.1 s to $10 \mathrm{~s}$, reduce the dilation factor from $k=8$ to $k=2$, and relax the occlusion threshold from $\tau=1 \%$ to $\tau=5 \%$.

These parameter changes add more votes that exhibit many of the mistakes from Figure 3, while also ensuring most points have a label. Critically, we could improve the quality of these features by removing some of the erroneous votes, but find it improves performance to use decoupled features despite their lower accuracy (Section 5.2). Decoupling teaches the network to correct mistakes, rather than pass through features. The result is a more useful function based on geometric reasoning. See Figure 4 for an example.

An additional consideration is how to use 2D image information to create input features for the 3D network. Because supervision is only available at certain points, it is important that the 3D convolutions learned on supervised points transfer to unsupervised points. We find, for example, that RGB features can actually damage performance because they do not exist at points unobserved by any color image. When we transfer RGB features to unobserved points by copying from nearest neighbors, they still look unrealistic and unlike the training set, so the network performs badly in those regions. Our choice is to aggregate only the final class label, encoded as a one-hot vector. This label is informative and compact enough to store. One-hot encodings transferred via nearest neighbor are more robust than RGB, because they are constant for the whole object. Most importantly, this label makes it difficult for the network to distinguish the pseudo-ground-truth points from other points. This property makes convolutions learned at supervised points generalize well and makes class labels an ideal choice for our setting.

\subsection{Sampling Diverse Scenes}

The third issue to address is how to sample training scenes from a large repository of data. In our system, a training scene is a short interval (10s) of data that typically includes a few million lidar points and a few dozen images. The question is which scenes should we use for training when the repository is orders of magnitude larger than our practical processing capacity?

The obvious answer is to sample scenes randomly. However, then we might get a training set filled only with roads and trees. It would be very unlikely to include rare objects, like phone booths or animals. Accordingly, our trained models would be biased towards the common classes.

There is a long tail of complex and rare scenes, and we would like to find and train on those instead. To do so, we use the pretrained 2D semantic segmentation network to estimate the objects in a scene, and then solve an optimization problem to select scenes containing images that provide a diverse training set. Specifically, we first randomly select a huge set of candidate images $\mathcal{I}$ and run the $2 \mathrm{D}$ network on them. For each image $I$, we check whether its semantic segmentation contains at least $p_{\min }$ pixels for each of the $|C|$ class labels, forming a binary vector $v_{I} \in\{0,1\}^{|C|}$. We then create a training set $S$ of exactly $N$ images by maximizing the following (negative energy) objective function:

$$
\max _{S \subset \mathcal{I}}\left\|\sum_{I \in \mathcal{S}} v_{I}\right\|_{\frac{1}{2}}^{\frac{1}{2}} \quad \text { s.t. }|S|=N
$$

This optimization aims to find as many examples of every class as possible, with quadratically diminishing returns per class. The set of scenes extracted from these images balances choosing individual classes that are rare with complex scenes that contain many objects. Note this approach is related to active learning [75, 9, 48, 2, 35], which has been used for segmentation datasets [46, 83, 89, 77, 40]. However, our case is distinct since our constraints come from processing an input example, not annotating it.

\section{Dataset}

To evaluate the proposed algorithms in our target setting, we curated a dataset containing lidar and posed images collected from car-mounted scanners in 20 cities spread across 15 countries on 5 continents (Figure 5). The scanning platform has 2 VLP16 lidar scanners mounted at oblique angles ( $\sim 45^{\circ}$ ) to scan building tops as well as street-level objects, and it has 7 color cameras with six in a hexagonal pattern and 1 pointing up (green lines in Figure 1).

Each of the 20 cities is divided into disjoint, interleaved sets of $2 \mathrm{~km} \times 2 \mathrm{~km}$ square regions, one set for training and 


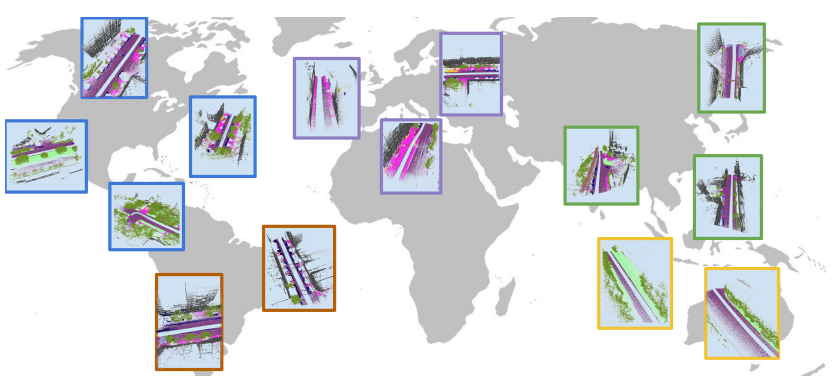

Figure 5: Our training and validation datasets include 3D scenes from cities around the world, a sampling of which are shown here.

another for validation. "Scenes" are sampled from these regions containing approximately ten seconds of data collection spanning approximately 1600 square meters with $3 \mathrm{M}$ lidar points and 60 color images on average.

The only training data available is a previously labeled set of 19,147 images captured in the training regions of the 20 cities along with 30 others. Those images were manually annotated with 44 categories representing different types of ground (road, sidewalk, driveway, crosswalk, etc.), vehicles (car, truck, bicycle, etc.), urban structures (building, bridge, fence, etc.), street-side objects (traffic signs, streetlights, etc.), and person. See Appendix E for a full list.

To produce a validation set for $3 \mathrm{D}$ semantic segmentation, we selected one scene randomly from each of the 20 cities and employed an interactive 3D labeling tool to identify one of the 44 semantic categories for every lidar point in the scene. This process took us several hours per scene using a highly optimized tool. For budgetary reasons, it was not practical for us to label a large set of training scenes with the same process, since it would cost millions of dollars.

When testing algorithms, we evaluate predicted 3D semantic segmentations on the validation set with mean class IoU (mIoU) following standard protocols [21]. Since the validation set has limited data, we are careful when computing aggregate mIoU statistics to avoid averaging in results of classes with very little data. Accordingly, we omit categories with less than 1000 points in each of at least 3 scenes when computing mean class IoU statistics. The remaining 26 classes include most with high interest to semantic mapping applications (e.g., building, road, sidewalk, crosswalk, car, truck, etc.) and $99.34 \%$ of all scanned points.

\section{Experimental Results}

Here, we run experiments to compare the proposed approach to baselines and to test the impact of each component of our approach. Unless otherwise specified, evaluations are performed on the 20 city dataset from Section 4.

\subsection{Comparisons to 2D Supervised Baselines}

Our first set of experiments evaluates the performance of the proposed methods in comparison to alternatives that

\begin{tabular}{|c|c|c|c|c|c|c|c|c|}
\hline Method & $\begin{array}{c}\text { Total } \\
\text { mIoU }\end{array}$ & $\begin{array}{c}\text { Mover } \\
\text { mIoU }\end{array}$ & Road & Tree & Bldg & Car & Person & Bike \\
\hline MVF & 48.1 & 43.0 & 84.8 & 88.8 & 91.6 & |46.6 & 19.9 & 54.7 \\
\hline Dense & 53.3 & 52.2 & 87.5 & 90.5 & 92.7 & 57.2 & 30.9 & 60.6 \\
\hline Ours & 59.5 & 68.9 & 91.5 & 92.5 & 94.3 & 83.1 & 48.8 & 70.8 \\
\hline
\end{tabular}

Table 1: Comparison to baseline methods. We report the mean as well as performance on the most common mover and non-mover classes. See Table 10 for results on all classes.

can work without direct 3D supervision. Since almost all prior methods are designed for benchmarks with 3D training sets, there are few baselines for our task. To the best of our knowledge, multi-view fusion is the only/best alternative [36]. We compare to that and to a baseline we created to represent a straightforward implementation of our approach with dense rather than sparse pseudo-labels.

- Multi-view fusion [36] (MVF): This approach is a standard implementation of multi-view fusion that aggregates semantic labels at 3D points based on voting of labels at pixels with unoccluded sight lines.

- Dense supervision (Dense): This approach performs multi-view fusion as described above and then trains a 3D network with dense supervision from the predicted labels. Although this baseline is a variant of our main idea rather than previous work, it is valuable to investigate how our approach of making pseudo-ground-truth compares to a simpler alternative.

For both our method and these baselines, we train a DeepLab v3 [13] with Xception65 [15] network on the labeled training images. That network takes in an RGB image and outputs a dense image prediction with a class label per pixel, which is used for multi-view fusion by the baselines and pseudo-supervision for our 3D network. For our 3D network, we use the SparseConv architecture [28].

Quantitative Comparisons. Table 1 compares the quantitative evaluation metrics for our method and the baselines. Overall, our method achieves $59.5 \mathrm{mIoU}$, while the best true baseline (MVFusion) achieves only 48.1. Our method also outperforms the alternative proposed that trains a 3D network with supervision from dense multi-view fusion (53.3 mIoU). The largest improvements are for mover classes, with a jump of $25.9 \mathrm{mIoU}$ over the MVFusion baseline. Our $3 \mathrm{D}$ network trained with sparse pseudo-supervision is able to better segment movers because it learns to discriminate classes based on their geometric shapes (e.g,. road vs. car).

Qualitative Comparisons. Figure 6 shows examples of semantic segmentations produced by our method and baselines. The other methods make errors that are clearly visible when looking at the 3D points (e.g. car labels on the ground and sidewalk). By contrast, our approach better accounts for both image and geometric features. 


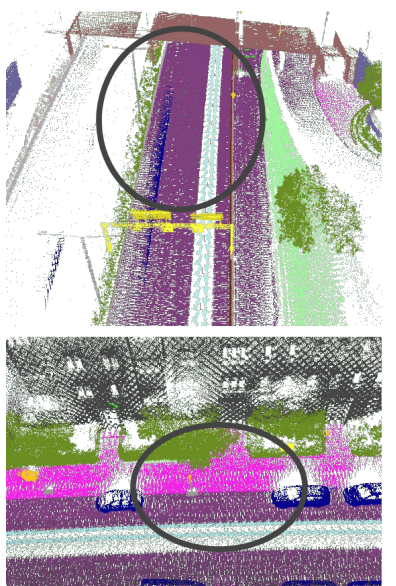

Ground Truth (Context)
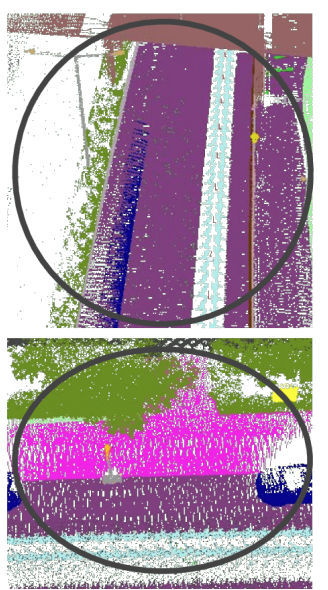

Ground Truth

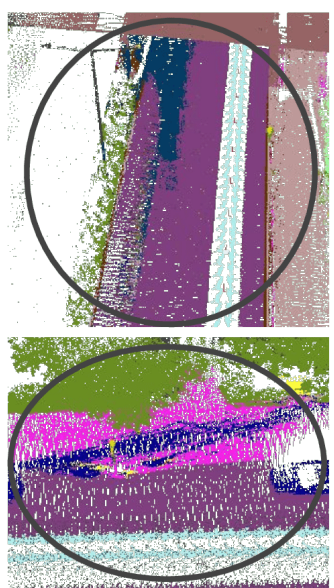

MVFusion

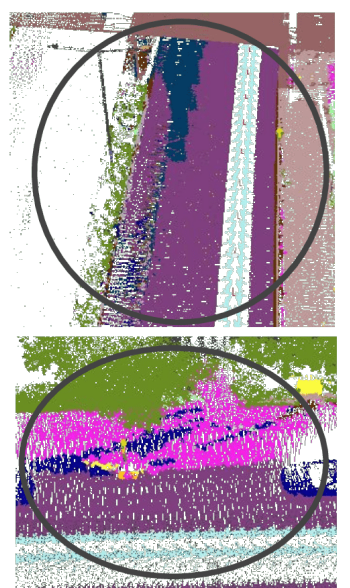

Dense Supervision
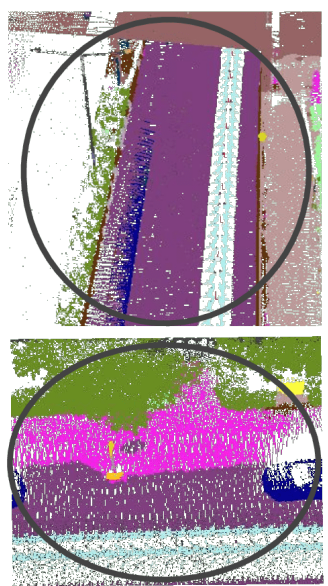

Ours

Figure 6: Qualitative results. Multiview fusion frequently makes nongeometric mistakes, especially for mover classes (votes for a bus end up on the ground, car and person on the sidewalk, building on person). Training a 3D network with dense supervision reduces these nongeometric mistakes slightly, but our approach improves the geometric prior substantially.

\begin{tabular}{l|cc}
\hline Method & $\begin{array}{c}\text { Total } \\
\mathrm{mIoU}(\uparrow)\end{array}$ & $\begin{array}{c}\text { Mover } \\
\mathrm{mIoU}(\uparrow)\end{array}$ \\
\hline Ours & 59.5 & 68.9 \\
\hline No Diverse Scene Sampling & 49.2 & 32.0 \\
No 3D Convolutions & 52.5 & 50.0 \\
No Sparse Supervision & 53.3 & 52.4 \\
No Timestamp Filtering & 53.4 & 62.9 \\
No Dilation & 59.8 & 68.5 \\
\hline
\end{tabular}

Table 2: Method ablations. All rows show variants of our method with a single algorithmic component removed or altered. Diverse scene sampling, 3D convolutions, timestamp filtering, and sparse supervision are all critical to achieving good results.

Typical errors for our method include 1) difficulty with classes that consistently differ between 2D and 3D, 2) missing small objects, and 3) failing to crisply separate $2 \mathrm{D}$ boundaries from badly fused features. For example, in Figure 6, top, the road behind the fence is mislabeled as fence due to consistently incorrect supervision in the sparse pseudo-ground-truth, and is only slightly improved over standard MVFusion.

\subsection{Ablation Studies}

Our second set of experiments investigates how each component of our system affects performance. Table 2 shows results of a knock-out study, where each row shows results of our method with one algorithmic component disabled. We find that diverse scene sampling, 3D convolutions, timestamp filtering, and sparse supervision are critical to achieving good results. Diverse scene sampling improves performance the most, by $10.6 \mathrm{mIoU}$, even when training on $250 \mathrm{~K}$ scenes and $750 \mathrm{~B}$ points. $3 \mathrm{D}$ convolution provides the second most improvement, as the semantic features before 3D convolution have all the problems shown in Figure 3. Dilating occluders helps with some classes (fences, trees, bicycles), but has mixed results overall.

In another experiment, we evaluate how import decoupling features is. We replace input features with pseudoground-truth values at pseudo-ground-truth locations. In other words, we increase coupling by improving feature quality while holding supervision fixed. As expected, increased coupling reduces performance- this ablation has increased train (pseudo-ground-truth) performance $(+8.8$ $\mathrm{mIoU}$ ) but reduced test (true) performance $(-2.6 \mathrm{mIoU})$. See Appendix B for more details.

\subsection{Experiments with the nuScenes Benchmark}

In this section, we evaluate our method using nuImages and the nuScenes lidarseg benchmark dataset [10]. While the nuScenes setting is different than ours (it has in-domain 3D supervision and data in only 2 cities), it enables several important experiments. In particular, it allows us to 1) evaluate how well our proposed architecture compares to the state-of-the-art (SOTA), 2) evaluate how well our approach performs when no, partial or full 3D supervision is available, and 3) study the generalization benefits of our 2D pseudo-supervision approach.

Is our 2D3DNet architecture SOTA? We first evaluate how our network architecture compares to previous work when given full 3D supervision. We trained the 2D3DNet architecture on the nuScenes lidarseg dataset, using nuImages to train our 2D network for features, and submitted our test split results to the nuScenes leaderboard. Our score is $80.0 \mathrm{mIoU}$, which is 1 st place (+1.7 mIoU) compared to all published methods, 3rd place overall including recent un- 


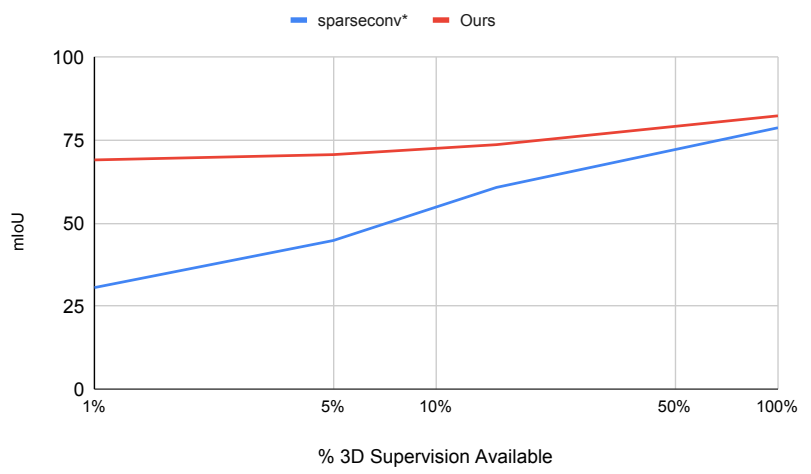

Figure 7: Compared to standard 3D baselines, our approach learns more quickly from a small amount of 3D data.

published submissions, and 1st place among all 2D-3D fusion based approaches (+2.3 mIoU). See Appendix A or the nuScenes lidarseg leaderboard for per-class mIoUs.

Is 2D supervision useful even when some 3D supervision is available? We next evaluate whether our pseudosupervision and 2D features can improve performance when combined with varying amounts of 3D supervision. We compare two approaches. The first approach is 2D3DNet, where some fraction of training scenes use 3D annotated labels and all remaining scenes use pseudo-supervision. All pseudo-supervision is generated by a $2 \mathrm{D}$ model trained on nuImages. The second approach is the 3D portion of our architecture ("SparseConv*") trained on 3D data. This SparseConv* baseline takes in surface normal features rather than our semantic features and is trained on 3D ground truth labels only; it is otherwise identical to our approach. Figure 7 provides mIoU results for several different sizes of the 3D training subset for both 2D3DNet (red) and SparseConv* (blue). We evaluate on the 11 classes present in both nuScenes and nuImages. The difference between the two curves suggests that 2D3DNet is not as dependent on a large $3 \mathrm{D}$ training set. At $0 \%$ supervision, 2D3DNet achieves 65.6 mIoU, versus $80.7 \mathrm{mIoU}$ at $100 \%$ supervision (92.0 vs 96.4 fwmIoU). By comparison, at 100\% 3D supervision SparseConv* reaches $78.8 \mathrm{mIoU}$, but only $30.6 \mathrm{mIoU}$ at $1 \%$ 2D3DNet's mIoU is 69.1 at $1 \%$. These results suggest that 3D supervision is superior when it is available in sufficient quantity, but that our methods for utilizing labeled 2D image collections can help 3D networks achieve competitive performance even when far less 3D supervision is available.

Does 2D supervision improve generalization? Finally, we evaluate whether our 2D pseudo-supervision improves generalization to new cities. We begin by partitioning the nuScenes lidarseg dataset into two subsets- Boston (467 scenes) and Singapore (383 scenes). We then consider three possible supervision strategies for training a 3D SparseConv* network. In the first case, we train using only 3D supervision from one city, and test generalization to the other city. In the second case, we train using 3D supervision from

\begin{tabular}{cc|cc}
\hline \multicolumn{2}{c}{ Supervision } & \multicolumn{2}{c}{ Validation } \\
\hline 3D & 2D & Same City & Other City \\
\hline Singapore & None & 70.3 & 38.9 \\
Singapore & Boston & 68.6 & $\mathbf{6 4 . 3}$ \\
None & Both & 63.1 & $\mathbf{6 4 . 3}$ \\
\hline Boston & None & 76.1 & 55.6 \\
Boston & Singapore & 74.8 & $\mathbf{6 8 . 8}$ \\
None & Both & 64.3 & 63.1 \\
\hline
\end{tabular}

Table 3: Adding our 2D pseudo-supervision helps SparseConv generalize better to cities for which there is no available 3D training data. The top three rows use Singapore as the 3D supervised city, and the bottom three use Boston. Rows 3 and 6 are the same model, since no 3D supervision is used in either case.

one city and also add pseudo-supervision from nuImages for the other city. In the final case, we train using no 3D supervision at all, and instead use our pseudo-supervision approach for both cities. The performance of each of the the three cases for each city are in Table 3.

We observe that: 1) Models trained only on 3D supervision from one city do not generalize well to the other city (38.9 Singapore $\rightarrow$ Boston, 55.6 Boston $\rightarrow$ Singapore). 2) 2D pseudo-supervision from both cities outperforms 3D supervision from a different city (64.3 vs 38.9 Boston, 63.1 vs 55.6 Singapore). 3) Adding 3D supervision from a different city on top of 2D pseudo-supervision improves performance only slightly (64.3 vs 64.3 Boston, 68.8 vs 63.1 Singapore). These observations suggest that our approach is a good alternative for 3D semantic segmentation in cities around the world for which 3D labeled data is not available.

\section{Conclusion}

In this paper, we investigate how to learn 3D semantic segmentation without any 3D labeled training data. We propose creating trusted pseudo-labels, leveraging $2 \mathrm{D}$ semantic predictions as input features to a 3D network, and efficiently sampling diverse scenes from a large unlabeled repository. These methods enable training 3D semantic segmentation models that are 6.2-11.4 mIoU better than baselines on a validation dataset sampled from 20 cities on five continents, and 16.7-25.9 mIoU better on mover classes. Further experiments with the nuScenes lidarseg dataset show that 2D3DNet outperforms published methods $(+1.7 \mathrm{mIoU})$ when given full supervision, our architecture is more robust than 3D supervised baselines when only a small amount of supervision is available $(+12.9-38.5 \mathrm{mIoU})$, and that pseudo-supervision helps with generalization to novel cities (+7.5-25.4 mIoU). These results suggest promising avenues for leveraging repositories of image data to train generalizable 3D models that can be deployed at scale. 


\section{Appendix}

This appendix contains additional information in support of the main paper. Specifically, it includes:

- Expanded supervised nuScenes results reporting the quantitative performance of 3D supervised models on the test and validation splits of the lidarseg task (Appendix A).

- Additional ablation studies investigating the impact of the key algorithms of the proposed method in more detail (Appendix B).

- Comparison to a SemanticKITTI 3D supervised model demonstrating the difficulty of transferring 3D supervision between datasets (Appendix C).

- Additional experiments on ScanNet investigating how the proposed approach can work without any indomain supervision at all (Appendix D).

- Additional baseline comparison statistics providing results for all 44 classes of the twenty city dataset (Appendix E).

- Additional qualitative visualizations showing the output of our model and fused semantic features (Appendix F).

- Implementation details for the 2D and 3D network architectures, multiview fusion, normal estimation, decoupling, optimization algorithm, and evaluation metrics (Appendix G).

\section{A. nuScenes Supervised Results}

In Table 4, we show the performance of our method on the test set (that is, we show the numbers available at www. nuscenes.org/lidar-segmentation on the nuScenes leaderboard). For all 16 classes, please see the leaderboard. To facilitate comparisons, we also provide our validation split numbers in Table 5 (no comparison to SPVCNN++ or GU-Net is possible because the details and code for those methods are unavailable, though we add a comparison to the SparseConv* architecture used for some experiments in Section 5.3). Our result is the highest published method on the test set, the 3rd highest overall (including recent unpublished submissions), and to our knowledge the highest reported on the validation split.

\section{B. Additional Ablation Studies}

In this section, we report results of experiments investigating ablations of our method.

How does feature decoupling affect performance? Table 6 demonstrates that feature decoupling improves performance. In this experiment, we use identical sparse supervision for both approaches. However, in the ablation (Abl),

\begin{tabular}{|c|c|c|c|c|}
\hline Method & $\mathrm{mIoU}$ & Pedestrian & Car & Bicycle \\
\hline SPVNAS [79] & 77.4 & 75.6 & 90.8 & 30.0 \\
\hline Cylinder3D++ [98] & 77.9 & 77.3 & 89.4 & 33.9 \\
\hline AF2S3Net [14] & 78.3 & 77.3 & 84.2 & 52.2 \\
\hline GU-Net & 80.3 & 81.3 & 91.4 & 33.4 \\
\hline SPVCNN++ & 81.1 & 83.4 & 92.2 & 43.1 \\
\hline LIFusion & 75.7 & 80.3 & 84.3 & 36.3 \\
\hline CPFusion & 77.7 & 78.1 & 86.2 & 37.0 \\
\hline Ours & 80.0 & 82.0 & 85.1 & 59.4 \\
\hline
\end{tabular}

Table 4: Results on the test split of the nuScenes lidarseg task [10]. Methods above the bar are lidar only; methods below the bar use both 2D and 3D reasoning. Methods in gray are currently unpublished and have no details besides the leaderboard description. We bold the highest published result. For up to date results on all 16 classes see the nuscenes leaderboard at nuscenes.org/lidar-segmentation (our entry is anonymized).

we improve the feature quality by using the pseudo-ground truth fusion where available. In other words, we ablate the decoupling so that the supervision remains the same but the feature quality improves. Despite more accurate features, the model performance is worse due to increased correlation between the features and the pseudo-ground truth.

What is the effect of 2D features? Table 7 shows results of a study investigating the performance of different features provided with $3 \mathrm{D}$ points as input to the 3D network. As expected, we find that it is critical to utilize the proposed semantic features derived from the 2D images (one-hot encoding of the semantic class generated by fusing predictions of the 2D network). Counter-intuitively, adding RGB channels as an additional feature hurts performance (by $1.7 \%$ $\mathrm{mIoU})$. We conjecture the reason is due to a test-time domain shift for lidar points not observed by any RGB image. For those points, we make our best effort to fill in missing RGB features by copying from nearest spatial neighbors. However, the resulting RGB features are out of domain for the pseudo-ground-truth points used for training, which are all observed directly by RGB images, leading to an adverse affect on performance. Yet, interestingly, the same is not true when using one-hot encodings of semantic labels. Since the network is trained with a protocol that decouples input semantic features from pseudo-ground-truth labels (Section 3.2), it learns to compensate for wrong semantic input labels and generalizes better to all points.

How effective is the diverse scene sampling? Table 8 shows more details on how the diverse scene sampling algorithm affects performance. For this study, we test training datasets with $250,2.5 \mathrm{~K}, 25 \mathrm{~K}$, and $250 \mathrm{~K}$ scenes, created both with and without our diverse scene sampling strategy (Section 3.3). We find that diverse sampling provides a sig- 


\begin{tabular}{|c|c|c|c|c|c|c|c|c|c|c|c|c|c|c|c|c|c|}
\hline Method & $\mathrm{mIoU}$ & 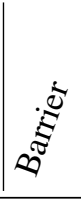 & S0 & $\stackrel{3}{0}$ & $\mathcal{U}^{\mathbb{\sigma}}$ & $\underbrace{\mathbb{E}}$ & $\frac{\overrightarrow{0}}{\stackrel{0}{0}}$ & $2^{\mathbb{E}}$ & $\tilde{0}^{\Xi}$ & 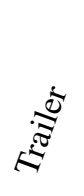 & 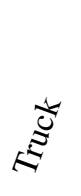 & 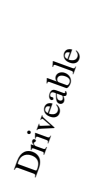 & 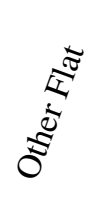 & 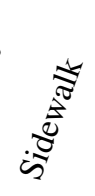 & : & 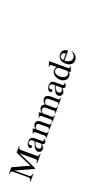 & 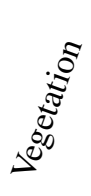 \\
\hline & & & & & & 30.2 & & 9.6 & & & & & 66.6 & 3.5 & & 83 & 79.8 \\
\hline & & .7 & & & & 35.1 & 7.5 & 1.3 & & & & 96.5 & 71.1 & 4.7 & 4.0 & 87.3 & 35.7 \\
\hline & & 74.8 & 34 & 85 & 88.4 & 42.2 & 72.4 & 72.2 & & & .5 & 96.0 & 70.8 & 71.2 & 1.5 & 86.7 & 84.4 \\
\hline & & 76.9 & 25.4 & 91.5 & 86 & 54.0 & 76.7 & 77.9 & 62.0 & .4 & 83.0 & 96.0 & 74.7 & 75.9 & 75.2 & 91.3 & 89.9 \\
\hline & 76.1 & 76.4 & 40.3 & 91.2 & 93.8 & 51.3 & 78.0 & 78.9 & 64.9 & & 84.4 & 96.8 & 71.6 & 76.4 & 75.4 & 90.5 & 87.4 \\
\hline Ours & 79.0 & 78.3 & 55.1 & 95.4 & 87.7 & 59.4 & 79.3 & 80.7 & 70.2 & 68.2 & 86.6 & 96.1 & 74.9 & 75.7 & 75.1 & 91.4 & 89.9 \\
\hline
\end{tabular}

Table 5: Results on the validation split of the nuScenes lidarseg task [10].

\begin{tabular}{|c|c|c|}
\hline Method & $\begin{array}{c}\text { All } \\
\text { Train Test }\end{array}$ & $\begin{array}{c}\text { Movers } \\
\text { Train Test }\end{array}$ \\
\hline Ours & $61.5 \mathbf{6 0 . 1}$ & \begin{tabular}{|l|l}
59.1 & 66.1
\end{tabular} \\
\hline Abl. & $70.3 \quad 57.5$ & 72.961 .0 \\
\hline
\end{tabular}

Table 6: Ablation of feature decorrelation. Train (pseudo-ground truth) mIoU increases and test (true) mIoU decreases.

\begin{tabular}{l|cc}
\hline Features & mIoU $(\uparrow)$ & Mover mIoU $(\uparrow)$ \\
\hline Semantic & $\mathbf{5 9 . 5}$ & $\mathbf{6 8 . 9}$ \\
Semantic + RGB & 57.8 & 65.8 \\
Normals & 27.7 & 21.8 \\
Normals + RGB & 30.6 & 26.9 \\
\hline
\end{tabular}

Table 7: Impact of different input features to the 3D network. Onehot encoding of the semantic class predicted by the 2D network is best. Semantic + Normals was also investigated but found to be unhelpful.

nificant performance improvement over random sampling at all dataset sizes (10.3\% mIoU on the largest dataset). The set of test scenes is sufficiently complex and varied that diverse training data improves generalizability and performance on multiple classes (other vehicle, motorcycle, street light, bicycle in particular). In contrast, the results achieved with a very large training dataset of random scenes $(250 \mathrm{~K}$ scenes) are not better than with a very small dataset (250 random scenes). We conjecture that it would require an impractically large training set of random scenes and a new loss carefully designed to handle extreme class imbalance to achieve comparable results to our diverse scene sampling strategy.

\section{SemanticKITTI Supervision Transfer}

In this experiment, we explore an alternative to $2 \mathrm{D}$ supervision- transferring the 3D supervision from an existing dataset. This alternate approach avoids many of the challenges discussed in Section 3.1. It also requires generalizing to a new terrestrial platform and to unseen cities,

\begin{tabular}{c|cccc}
\hline Method & \multicolumn{4}{c}{ mIoU } \\
\hline \# Scenes & 250 & $2.5 \mathrm{~K}$ & $25 \mathrm{~K}$ & $250 \mathrm{~K}$ \\
\hline Random Sampling & 53.1 & 50.8 & 51.3 & 49.2 \\
Diverse Sampling & 58.1 & 58.3 & 58.7 & 59.5 \\
\hline
\end{tabular}

Table 8: Our approach makes efficient use of large unsupervised data sets. By contrast, random sampling performs worse at all dataset sizes and does not reliably improve performance as the dataset grows (rare cases remain rare and difficult to learn).

\begin{tabular}{c|c|ccccccc}
\hline & Mean & Bld. & Road & Car & SW. Fence & Pole & Bike \\
\hline Ours & $\mathbf{7 2 . 7}$ & $\mathbf{9 6 . 1}$ & $\mathbf{9 3 . 3}$ & $\mathbf{8 4 . 0}$ & $\mathbf{7 7 . 8}$ & $\mathbf{6 0 . 7}$ & $\mathbf{6 0 . 7}$ & $\mathbf{7 5 . 8}$ \\
{$[7,98]$} & 24.0 & 76.3 & 38.9 & 68.5 & 28.4 & 18.2 & 12.9 & 10.1 \\
\hline
\end{tabular}

Table 9: A comparison to a state of the art 3D model (Cylinder3D [98]) trained with 3D supervision on SemanticKITTI [7].

countries, and continents.

Table 9 shows a comparison to a state of the art model, Cylinder3D [98], trained with 3D supervision from SemanticKITTI [7] and tested on our twenty city dataset. We choose SemanticKITTI because of its class overlap with our evaluation set. To adapt our input scans as directly as possible, we provide the pair of full LiDAR sweeps as inputs to their network for each scan. To provide a fair evaluation, we consider only classes shared between the two evaluation sets, and we report only the IoU of classes with the best performance for [98] in Table 9. Not surprisingly, this simple baseline is not competitive with our approach due to differences in the sensor patterns (different lidar angles) and scene content ( 1 training city versus 20 evaluation cities). Yet, the large performance gap (48.7 points) highlights the problems of domain adaptation on $3 \mathrm{D}$ point clouds.

\section{Application to Indoor Datasets (ScanNet)}

As an experiment to test the generality of our main ideas under extreme settings, we ran an experiment on the ScanNet Semantic Segmentation Benchmark [21] using only 3D point cloud inputs and no ground truth data from ScanNet 


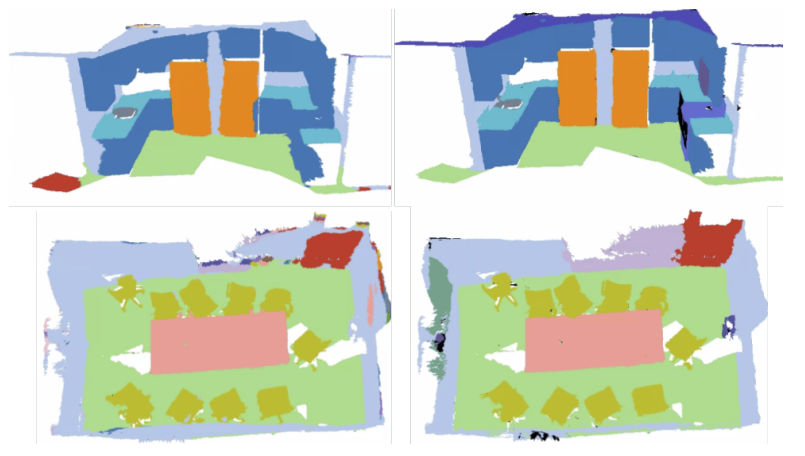

Figure 8: ScanNet 3D segmentations using our network trained only from MSeg images (no ScanNet data at all) and tested only with 3D point inputs (no images from the test scenes). The 3D predictions (left) are originally for the 194 MSeg classes; we map onto the 20 ScanNet classes for display purposes to compare to the ground truth (right).

at all (no 3D point labels, no image labels, and not even the ScanNet category list). To train our 3D network, we used pseudo-supervision derived from a pretrained MSeg Universal 2D model [53] (which was trained on several 2D datasets, not including ScanNet). Specifically, we predicted semantic segmentations of images from the ScanNet training set using the MSeg model, ran our algorithms to create sparse pseudo-ground-truth from those segmentations, trained our 3D model, and tested it on inputs that include only 3D points (using 3D normals as input features), and then evaluate the result using an approximate mapping between MSeg and ScanNet classes. The result is $42.6 \mathrm{mIoU}$ on the ScanNet validation set. Example results are shown in Figure 8. Of course, these results are not as good as methods trained with supervision from either 2D or 3D ScanNet data. However, it is remarkable that it can perform so well (9 points better than PointNet++ [71] trained with full 3D supervision from ScanNet) when the input at inference is only $3 \mathrm{D}$ geometry, and the supervision at training comes only from labeled 2D image collections from other domains, in spite of an ambiguous mapping from MSeg to ScanNet classes.

\section{E. Expanded Baseline Comparison Statistics}

Table 10 reports full quantitative statistics for the baseline comparison experiment described in Section 5.1 (i.e., it is an expanded version of Table 1). In addition to reporting per-class IoU for all 44 classes in the twenty city dataset, it also reports the numbers of points and GT scenes per class, which were used to determine the set of 26 classes for computing the mIoU in Table 1 (as described in Appendix G.5). We also provide a visualization from one ground truth scene for illustrative purposes in Figure 9.

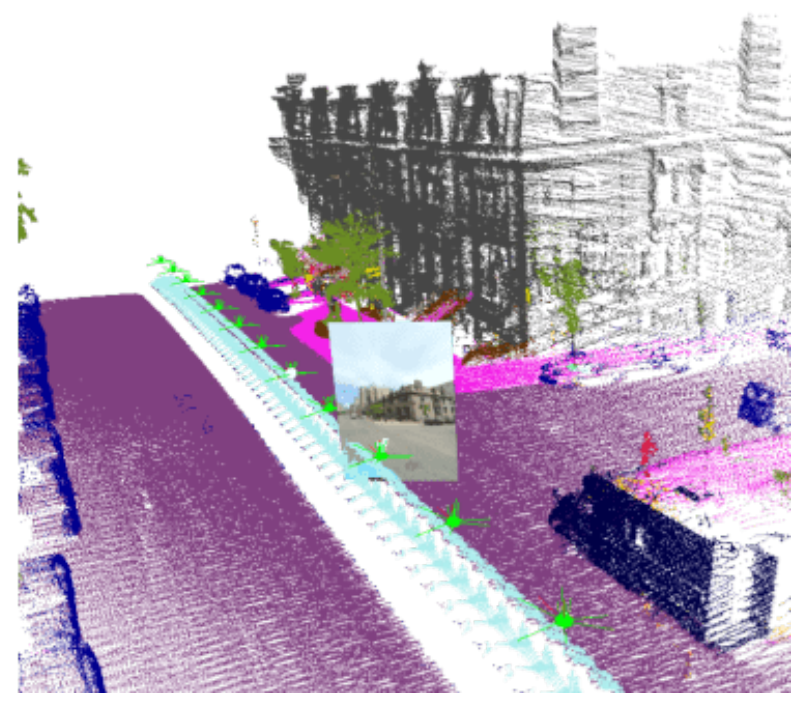

Figure 9: Our input "scenes" contain a sequence of RGB images from 7 cameras operating at $\sim 2 \mathrm{~Hz}$ (camera extrinsics shown as bright green lines), and two independent lidar sensors capturing 16 beam sweeps at $\sim 10 \mathrm{~Hz}$.

\section{F. Additional Qualitative Results}

In Figure 10, we provide additional qualitative visualizations of our method on our 20-city evaluation set. For each, we provide a ground truth visualization for context, as well as close-up comparisons of our intermediate multiview fusion predictions (as generated by Algorithm 1 with the hyperparameters described in Section 3.1), our final output predictions, and the ground truth.

\section{G. Implementation Details}

In this section, we provide low level implementation details required for full reproducibility. We discuss network architecture and optimizer details, normal and radii estimation, multiview fusion, optimization details, and our evaluation procedure.

\section{G.1. Fusion Details}

The important aspects of our multiview fusion algorithm are described in Section 3.1. We describe the lower level implementation details here with the goal of ensuring reproducibility. Most of the details that are omitted in Section 3.1 exist to improve runtime efficiency (e.g., the addition of a kdtree, the specific order of correspondence checks to avoid unncessary projections). There is also one additional parameter, $\Delta t_{\text {thresh }}$, which is set to 20 seconds in all cases. When a point is about to be rasterized into a depth image as a surfel, the point-image time difference is compared to $\Delta t_{\text {thresh }}$; points exceeding the threshold are not rasterized into the image. This parameter, too, exists primarily for performance reasons- typically it only affects the resulting 

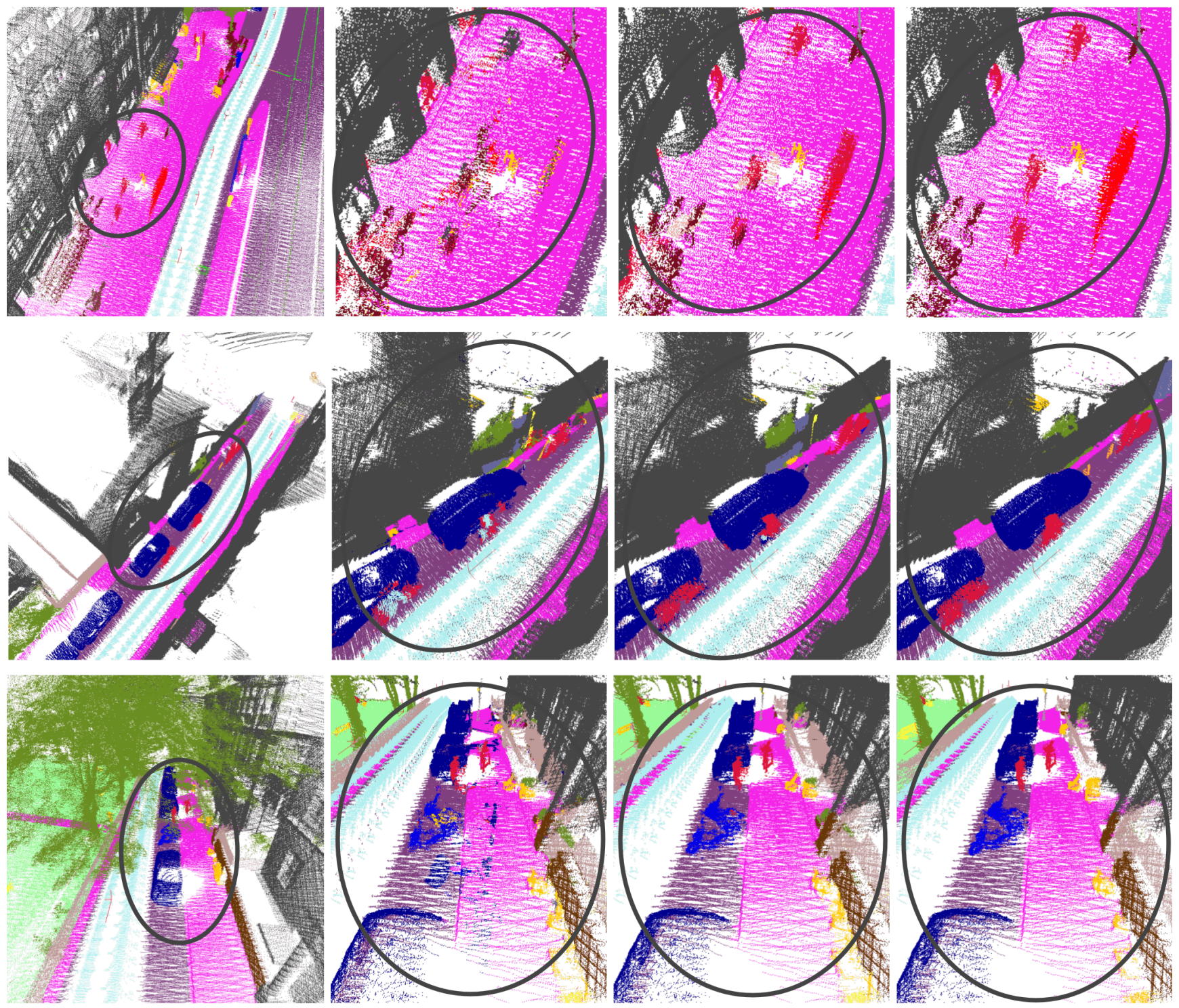

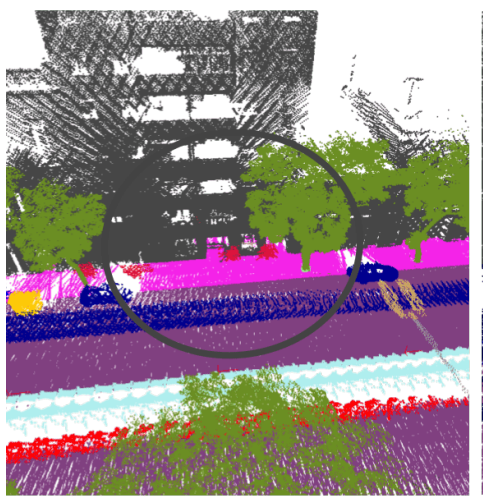

Ground Truth (Context)

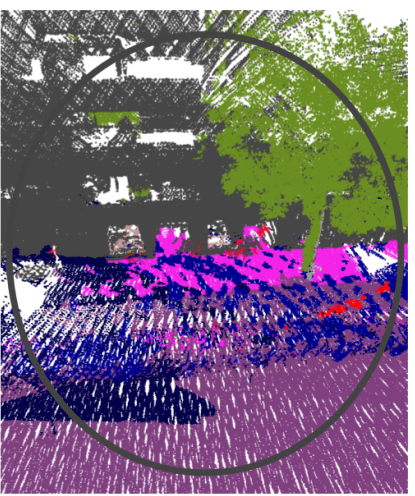

MVFusion (Alg. 1)

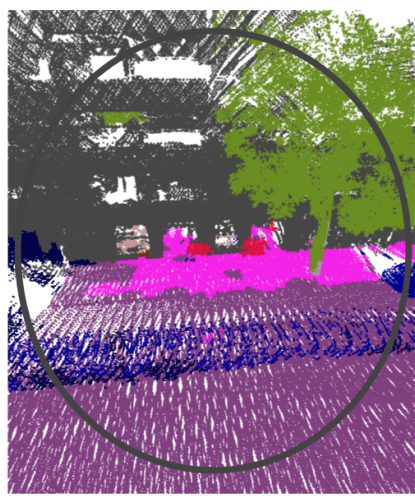

Ours

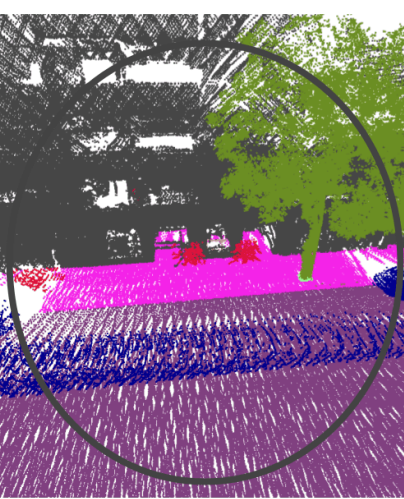

Ground Truth

Figure 10: Qualitative visualizations on lidar scenes from our 20-city evaluation set. We visualize our first-stage dense label aggregations, the final output labels, and the ground truth predictions. 


\begin{tabular}{|c|c|c|c|c|c|}
\hline Class & MVFusion & Dense Supervision & Ours & \# GT Points & \# GT Scenes \\
\hline SELF & 97.5 & 97.6 & 98.7 & $4,135,276$ & 20 \\
\hline BUILDING & 91.6 & 92.7 & 94.3 & $18,430,607$ & 17 \\
\hline TREE & 88.8 & 90.5 & 92.5 & $12,976,114$ & 19 \\
\hline PAVED_ROAD & 84.8 & 87.5 & 91.5 & $13,030,871$ & 20 \\
\hline SIDEWALK & 65.3 & 68.6 & 72.6 & $4,356,483$ & 18 \\
\hline CROSSWALK & 48.9 & 56 & 61.8 & 317,971 & 4 \\
\hline DRIVEWAY & 23 & 28.4 & 25.2 & 333,199 & 6 \\
\hline TERRAIN & 70.6 & 72.3 & 75.6 & $3,489,174$ & 16 \\
\hline OTHER_STRUCTURE & 35 & 39.5 & 42.9 & 373,576 & 12 \\
\hline FENCE & 42.1 & 45.5 & 47.2 & 818,815 & 15 \\
\hline WALL & 25.6 & 32.7 & 33.5 & 384,525 & 9 \\
\hline GUARD_RAIL & 31.2 & 33.9 & 33.9 & 113,600 & 3 \\
\hline TRAFFIC_SIGN & 43.2 & 49.7 & 58.7 & 56,779 & 10 \\
\hline BRIDGE & 88.1 & 90 & 90.7 & 923,639 & 3 \\
\hline STREET_LIGHT & 50.2 & 59.3 & 66.8 & 14,355 & 4 \\
\hline BUSINESS_SIGN & 9.2 & 23.5 & 27.3 & 26,630 & 3 \\
\hline OTHER_PERMANENT_OBJECT & 21.9 & 19.7 & 20.5 & 77,679 & 12 \\
\hline OTHER_TEMP_OBJECT & 40.1 & 43.3 & 48 & 353,521 & 15 \\
\hline CAR & 46.6 & 57.2 & 83.1 & $1,543,843$ & 19 \\
\hline TEMP_TRAFFIC_SIGN & 2.7 & 1.4 & 0 & 6,836 & 3 \\
\hline TRUCK & 57.9 & 63.8 & 78.9 & $1,898,201$ & 10 \\
\hline MOTORCYCLE & 32.1 & 38.8 & 51.2 & 31,537 & 4 \\
\hline POLE & 31.4 & 39.1 & 51.7 & 152,212 & 20 \\
\hline PERSON & 19.9 & 30.9 & 48.8 & 123660 & 10 \\
\hline OTHER_VEHICLE & 47.1 & 62.1 & 80.4 & 15014 & 3 \\
\hline BICYCLE & 54.7 & 60.6 & 70.8 & 72763 & 4 \\
\hline SKY & - & - & - & 0 & 0 \\
\hline TUNNEL & - & 0 & 0 & 0 & 0 \\
\hline BUS_STOP & 2.8 & 2.4 & 0 & 34,856 & 1 \\
\hline PHONE_BOOTH & 0 & 0 & 0 & 3,349 & 1 \\
\hline UNPAVED_ROAD & 0 & 0 & 0 & 0 & 0 \\
\hline TRAFFIC_LIGHT & 31 & 36.8 & 53.4 & 2,972 & 1 \\
\hline OTHER_GROUND & 0 & 0 & 0 & 554 & 0 \\
\hline PARKING_METER & 0.1 & 0 & 0 & 4,039 & 1 \\
\hline MAILBOX & 20.3 & 13.5 & 0 & 2,659 & 1 \\
\hline FIRE_HYDRANT & 50.7 & 4.6 & 0 & 2,112 & 1 \\
\hline BILLBOARD & 0 & 0 & 0 & 4,424 & 2 \\
\hline TEMP_CONE & 18.1 & 25.4 & 0 & 4,554 & 1 \\
\hline BUS & 0 & 0 & 0 & 0 & 0 \\
\hline VEHICLE_ON_RAILS & 0.3 & 0 & 0 & 887 & 0 \\
\hline MOUNTAIN & 0 & 0 & - & 0 & 0 \\
\hline ANIMAL & 0 & 0 & 0 & 141 & 0 \\
\hline WATER & 0 & 0 & - & 0 & 0 \\
\hline RIDER & 0.2 & 0.3 & 5.8 & 30606 & 2 \\
\hline
\end{tabular}

Table 10: Quantitative results on the full set of 44 classes. For each class, we also report the number of ground truth points and the number of ground truth scenes containing at least 1,000 points. The criterion for inclusion in the evaluation in Table 1 was at least 3 scenes with at least 1,000 points each (note some classes have tens of millions of points across 15+ scenes). Classes above the bar were included in evaluation. 
images when the car is still for long periods of time and there is extraneous data that does not need to be rendered to get a reasonable occlusion map ( $\Delta t_{\text {thresh }}$ does this culling). All other parameters are described in Section 3.1. Our algorithm is implemented in $\mathrm{C}++$ and pseudocode is available in Algorithm 1.

Fusion parameters for our dataset are given in Section $3.1\left(d_{\max }=400\right)$. For most nuScenes experiments, hyperparameters are largely unchanged, though we adjust the supervision $\Delta t_{\max }$ to 0.085 to account for the different image sampling rate in nuScenes compared to our dataset (12 vs $2 \mathrm{hz}$ ). Before submitting to the leaderboard, we did additional experiments with 3D supervision to determine more optimal feature parameters when decoupling from 2D supervision is not a factor. We found that reducing $d$ to 1.5 , increasing $\tau$ to $10 \%$, and adding an additional term to $w_{i j}$ to scale inversely with class rarity (i.e., divide by the fraction of lidar points in the train split for that class) improved performance slightly, because they enable the network to better identify and correctly classify small and rare classes. These changes were made only for the leaderboard submission.

\section{G.2. Network Architecture Details}

In this section, we provide details regarding the 2D3DNet network architecture used in our experiments, for reproducibility purposes.

3D Architecture Our 3D network architecture is a 3D Sparse Conv Network [28]. Each encoder layer $(A, B)$ consists of a $3 \times 3 \times 3$ spatial convolution with $A$ output features, followed by a $3 \times 3 \times 3$ spatial convolution with $B$ output features, followed by a $2 \times 2 \times 23 \mathrm{D}$ maxpool operation. Each convolution is first normalized normalized by dividing by the activation of an occupancy feature convolution. Then batch norm is applied, followed by relu. The encoder layers are: $(64,64),(64,96),(96,128),(128,160),(160,192)$, $(192,224),(224,256)$. The encoder layers are followed by a bottleneck layer. The bottleneck layer is $(256,256)$ and mirrors the encoder layers (the only difference is there is no maxpool after the convolution block). Finally there are decoder layers. Each decoder layer $(A, B)$ consists of a $2 \times 2 \times 2$ unpooling, followed by a $3 \times 3 \times 3$ spatial convolution with $A$ output features, followed by another $3 \times 3 \times 3$ spatial convolution with $B$ output features. As before, each convolution is followed by relu and then by dividing by the activation of an occupancy feature convolution for normalization. The decoder layers are: $(256,256),(224,224),(192,192)$, $(160,160),(128,128),(96,96),(64,64)$. Finally we apply a sequence of three $3 \times 3 \times 3$ spatial convolution layers. The layer sizes are (64, 64, output_channel_count). The first two convolutions are followed by normalization, batch norm, and relu, as usual, while the last convolution is followed only by normalization. Finally we apply a softmax function to get probabilities (we use a softmax cross en-

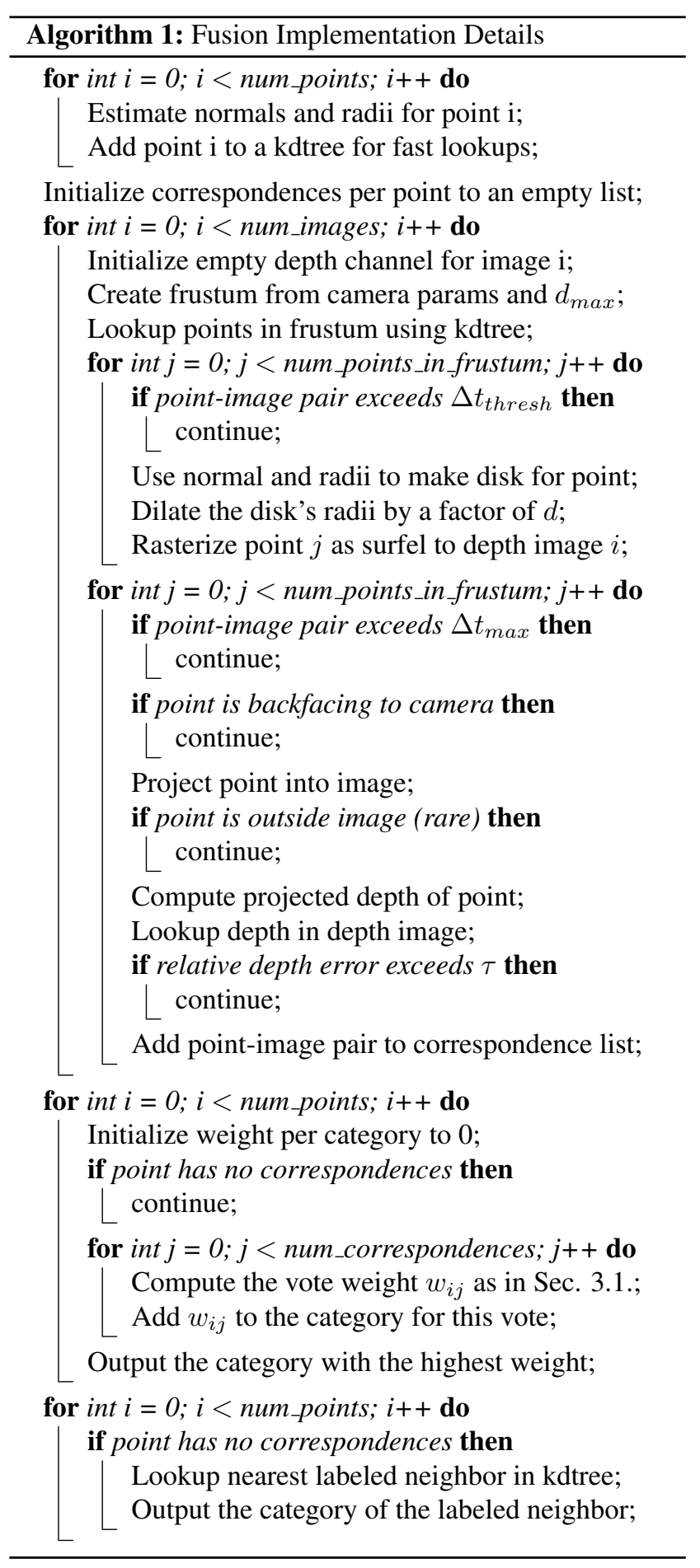

tropy loss). The loss is applied to voxels. For inference, we project back to points. At training time, we pad to 120,000 voxels. We randomly sample $1 \mathrm{M}$ points from the scene for input to the network for memory reasons. Where not otherwise specified, the input features are a one-hot encoding of 
the predicted semantic class per point (no other features).

3D Optimizer and Data Augmentation Details We use a momentum optimizer with 0.9 momentum and an initial learning rate of 0.03 . We use this learning rate for $200 \mathrm{~K}$ steps. After $200 \mathrm{~K}$ steps, we begin a cosine decay from $200 \mathrm{~K}$ steps to $700 \mathrm{~K}$ steps, at which point the learning rate fully decays to 0 and training is complete. The loss is softmax cross entropy classification loss plus a weight decay of 0.0001 . We use $20 \mathrm{~cm}$ voxels and a batch size of 8 . For data augmentation, we apply the following at training time only: random crops of 3 square meters, $x$ and y rotations of up to +-10 degrees, $\mathrm{z}$ rotations of up to +-180 degrees, and a random scale factor between 0.9 and 1.1.

3D Details (nuScenes) For nuScenes, the 3D pipeline is identical to our dataset except for the following specific changes. First, the voxel size is reduced from $20 \mathrm{~cm}$ to $5 \mathrm{~cm}$, training time voxel padding is increased from 120,000 to 240,000 , and the batch size is reduced from 8 to 3 . Second, no random crops, rotations, or scales are done (i.e., we skip the data augmentation). Finally, we train for only $450 \mathrm{~K}$ steps rather than $700 \mathrm{~K}$; there is still a $200 \mathrm{~K}$ warmup period with the same learning rate, but the cosine decay schedule is accelerated to reach 0 at $450 \mathrm{~K}$. Note that in addition, when doing multiview fusion on nuScenes we find and cull ego vehicle points when making the depth images, as otherwise the ego vehicle unnecessarily occludes a portion of the final image.

2D Architecture Our 2D network architecture is DeepLabv3 [13] with Xception [15]. For our dataset, we use Xception65 and source images downsampled to a resolution of $1152 \times 768$. For nuImages, we use Xception71 and images of resolution $1600 \times 900$. We pretrain the network on ImageNet classification [74]. On nuImages we optimize with a batch size of 32 , a backbone output stride of 16 , a decoder output stride of 4 , a base learning rate of $0.0045,150 \mathrm{~K}$ total steps, atrous rates of 6,12 , and 18 , apply a hard pixel mining loss on the top $0.25 \%$ of pixels, and set a last layer gradient multiplier of 10 .

\section{G.3. Normal and Radii Estimation}

Here, we describe our approach to estimating surface normals and radii per lidar point. Many other methods of normal estimation would likely be fine, especially since their primary use in our pipeline is to create depth maps. We chose this particular approach because we found it generated high quality results on our data. Conceptually, we estimate a coordinate frame per point by doing a PCA decomposition on a neighborhood around the point, growing the neighborhood until the PCA decomposition is nondegenerate. Then the tangent, bitangent, and normal are the first, second, and third principal axes, respectively. Radii along the tangent and bitangent directions are set to a fixed fraction of the size of the neighborhood needed to get a stable decomposition. This defines a surface disk (surfel) parameterized by a normal, two radii, and a tangent. Implementation details are below.

Consider one lidar point. First, we start by creating a neighborhood around the point with radius $25 \mathrm{~cm}$. Next, we use a kdtree to gather all points in the neighborhood. If there are too many points $(>32)$, we randomly subsample until there are at most 32. We then do a PCA decomposition of the remaining points centered at the point in question. If there are not enough points to do a PCA decomposition $(<3)$ or the PCA is degenerate, we double the neighborhood radius and try again, up to a maximum radius of $2 \mathrm{~m}$ (if there are still not enough points for a nondegenerate PCA decomposition, we give up and simply set the normal to the world up vector, though this is exceedingly rare in practice). We determine degeneracy for the decomposition by asserting that the first two principal standard deviations are at least $10 \%$ of the neighborhood size, clipped to the narrow range $[.25 \mathrm{~cm}, 1 \mathrm{~cm}]$. Once the PCA is determined nondegenerate, it defines a coordinate frame around the point based on the orthogonal directions of maximum variance. The direction of maximum variation is chosen as the tangent, and the radius in the tangent direction is set to $0.25 *$ the neighborhood size. The second radius, the radius in the bitangent direction, is set to the aspect ratio * the first radius. The aspect ratio is determined as the ratio of the standard deviations of the first two principal axes (i.e., $\sqrt{\frac{\sigma_{1}}{\sigma_{0}}}$ ).

\section{G.4. Optimization Details}

In this section, we report details for the optimization algorithm described in Section 3.3. The problem is to solve:

$$
\max _{S \subset \mathcal{I}}\left\|\sum_{I \in \mathcal{S}} v_{I}\right\|_{\frac{1}{2}}^{\frac{1}{2}} \quad \text { s.t. }|S|=N
$$

Before beginning the optimization, we compute terms $v_{I}$. For each image, we run our pretrained $2 \mathrm{D}$ conv network. We compute a histogram of class frequencies, and set $v_{I, c}:=1$ if at least $2 \%$ of the predicted pixels for $I$ are class $c$, and 0 otherwise. Once $v_{I}$ is computed for all images, we run the optimization. There are 1.04 billion images in the candidate set, so efficiency is critical. In order to make it tractable, we separate the optimization into two phases. First, we filter out images that do not contain at least one fairly rare class. This removes the vast majority of images while being unlikely to substantially affect the final solution quality; it also makes it possible to fit all remaining $v_{I}$ in memory. The second step is a greedy algorithm. We start with an empty set, and repeatedly iterate through the remaining set of candidate images, choosing the image that most improves the current solution. At each step we 
add the chosen image to the current solution, and recompute the current overall energy. Then, we iterate over each image and compute the affected partial terms of each remaining image's nonzero categories with respect to the objective. Once we have reach $|S|=N$, the optimization ends. This approach is not guaranteed to give an optimal result, but is necessary to accommodate the large number of image vectors.

\section{G.5. Evaluation Details}

In this section, we provide details about how ground truth labels were acquired and used for evaluation in the twenty city dataset.

Our 2D image collection has labels for 44 classes. There are no currently available $3 \mathrm{D}$ semantic labels. In order to evaluate our method in 3D, we annotate 20 scenes (approximately 50 million LiDAR points), directly adapting the class definitions from 2D. As described in Section 4, there are some differences in 2D versus 3D labeling standards. For example, in the 2D dataset, electrical wires were not labeled; we label wires separately and do not evaluate at those points.

Labeling the classes in 3D for evaluation poses some challenges. Some of the classes, such as vehicle on rails, are quite rare and therefore unlikely to appear repeatedly in a small number of scenes. Some classes, such as street light (which contains only the bulb, not the attached pole), are small. This makes them more difficult for a human to reliably find and segment accurately, and increases the effect of noisy boundaries on the metrics. Some classes, like animal or parking meter, are both small and rare, compounding these issues. Finally, some classes (e.g. sky, water) do not appear at all in the 3D point cloud. Therefore, in Section 5.1 we only report results for the 21 classes that appear in at least three different scenes with at least 1,000 points in each scene. This is $99.34 \%$ of all scanned points.

In total, we drop $0.66 \%$ of the 50 million points from the evaluation when reporting the mean IoU, most of which are large objects from classes that appear rarely (e.g., bridge). The classes that are kept versus those that are removed are provided in Table 10.

It is important to note that while the labels that are thrown out are less reliable than those used for evaluation, not all methods perform equally well on the noisy classes. One important bias is that our 3D network is based on sparse voxel convolutions with a resolution of $20 \mathrm{~cm}^{3}$. As a result, our method is not well suited for objects that are small compared to this resolution. Fusion approaches are comparatively less affected by small objects. An extreme case is the fire hydrant class. This class accounts for only $0.004 \%$ of $3 \mathrm{D}$ points, but our MVFusion approach still achieves an IoU of 60.4; however, all tested methods based on sparse voxel convolutions achieve an IoU of zero, a difference well in ex- cess of the noise in the GT for this class. In order to handle these classes robustly, it would be necessary to adjust the 3D approaches to compensate for this deficiency.

\section{References}

[1] Idan Achituve, Haggai Maron, and Gal Chechik. Selfsupervised learning for domain adaptation on point clouds. In Proceedings of the IEEE/CVF Winter Conference on Applications of Computer Vision, pages 123-133, 2021. 2

[2] Hamed H Aghdam, Abel Gonzalez-Garcia, Joost van de Weijer, and Antonio M López. Active learning for deep detection neural networks. In Proceedings of the IEEE/CVF International Conference on Computer Vision, pages 36723680, 2019. 5

[3] Inigo Alonso, Luis Riazuelo Montesano, Ana C Murillo, et al. Domain adaptation in lidar semantic segmentation. arXiv preprint arXiv:2010.12239, 2020. 2

[4] Humam Alwassel, Dhruv Mahajan, Bruno Korbar, Lorenzo Torresani, Bernard Ghanem, and Du Tran. Self-supervised learning by cross-modal audio-video clustering. Advances in Neural Information Processing Systems, 33, 2020. 3

[5] Iro Armeni, Zhi-Yang He, JunYoung Gwak, Amir R. Zamir, Martin Fischer, Jitendra Malik, and Silvio Savarese. 3d scene graph: A structure for unified semantics, 3d space, and camera. In Proceedings of the IEEE International Conference on Computer Vision, 2019. 2

[6] Yusuf Aytar, Carl Vondrick, and Antonio Torralba. Soundnet: Learning sound representations from unlabeled video. In Advances in neural information processing systems, pages 892-900, 2016. 3

[7] Jens Behley, Martin Garbade, Andres Milioto, Jan Quenzel, Sven Behnke, Cyrill Stachniss, and Jurgen Gall. Semantickitti: A dataset for semantic scene understanding of lidar sequences. In Proceedings of the IEEE International Conference on Computer Vision, pages 9297-9307, 2019. 1, 2, 10

[8] M. Bredif, D. Boldo, M. Pierrot-Deseilligny, and H. Maitre. $3 \mathrm{~d}$ building reconstruction with parametric roof superstructures. International Conference on Image Processing, 2007, 2:537-540, 2007. 2

[9] Samuel Budd, Emma C. Robinson, and Bernhard Kainz. A survey on active learning and human-in-the-loop deep learning for medical image analysis. Medical Image Analysis, 71:102062, 2021. 5

[10] Holger Caesar, Varun Bankiti, Alex H Lang, Sourabh Vora, Venice Erin Liong, Qiang Xu, Anush Krishnan, Yu Pan, Giancarlo Baldan, and Oscar Beijbom. nuScenes: A multimodal dataset for autonomous driving. In Proceedings of the IEEE/CVF conference on computer vision and pattern recognition, pages 11621-11631, 2020. 1, 2, 7, 9, 10

[11] Erzhuo Che, Jaehoon Jung, and Michael J Olsen. Object recognition, segmentation, and classification of mobile laser scanning point clouds: A state of the art review. Sensors, 19(4):810, 2019. 2

[12] Jie Chen and Baoquan Chen. Architectural modeling from sparsely scanned range data. Int. J. Comput. Vision, 78(23):223-236, 2008. 2 
[13] Liang-Chieh Chen, George Papandreou, Florian Schroff, and Hartwig Adam. Rethinking atrous convolution for semantic image segmentation. arXiv preprint arXiv:1706.05587, 2017. 6, 15

[14] Ran Cheng, Ryan Razani, Ehsan Taghavi, Enxu Li, and Bingbing Liu. 2-s3net: Attentive feature fusion with adaptive feature selection for sparse semantic segmentation network. In Proceedings of the IEEE/CVF Conference on Computer Vision and Pattern Recognition, pages 12547-12556, 2021. 9

[15] François Chollet. Xception: Deep learning with depthwise separable convolutions. In Proceedings of the IEEE conference on computer vision and pattern recognition, pages 1251-1258, 2017. 6, 15

[16] Christopher Choy, JunYoung Gwak, and Silvio Savarese. 4d spatio-temporal convnets: Minkowski convolutional neural networks. In Proceedings of the IEEE Conference on Computer Vision and Pattern Recognition, pages 3075-3084, 2019. 2

[17] Christopher Choy, Jaesik Park, and Vladlen Koltun. Fully convolutional geometric features. In Proceedings of the IEEE International Conference on Computer Vision, pages 8958-8966, 2019. 2

[18] S. Clode, P. Kootsookos, and F. Rottensteiner. The automatic extraction of roads from lidar data. In Proceedings of American Society of Photogrammetry and Remote Sensing, volume 35, pages 231-237. ASPRS, May 2004. 2

[19] Marius Cordts, Mohamed Omran, Sebastian Ramos, Timo Rehfeld, Markus Enzweiler, Rodrigo Benenson, Uwe Franke, Stefan Roth, and Bernt Schiele. The cityscapes dataset for semantic urban scene understanding. In Proceedings of the IEEE conference on computer vision and pattern recognition, pages 3213-3223, 2016. 2

[20] Tiago Cortinhal, George Tzelepis, and Eren Erdal Aksoy. Salsanext: Fast, uncertainty-aware semantic segmentation of lidar point clouds for autonomous driving. arXiv preprint arXiv:2003.03653, 2020. 2, 10

[21] Angela Dai, Angel X Chang, Manolis Savva, Maciej Halber, Thomas Funkhouser, and Matthias Nießner. Scannet: Richly-annotated $3 \mathrm{~d}$ reconstructions of indoor scenes. In Proceedings of the IEEE Conference on Computer Vision and Pattern Recognition, pages 5828-5839, 2017. 6, 10

[22] Angela Dai and Matthias Nießner. 3dmv: Joint 3d-multiview prediction for $3 \mathrm{~d}$ semantic scene segmentation. In Proceedings of the European Conference on Computer Vision (ECCV), pages 452-468, 2018. 2

[23] Ariel Ephrat, Inbar Mosseri, Oran Lang, Tali Dekel, Kevin Wilson, Avinatan Hassidim, William T. Freeman, and Michael Rubinstein. Looking to listen at the cocktail party: A speaker-independent audio-visual model for speech separation. ACM Trans. Graph., 37(4), July 2018. 3

[24] C. Frueh, S. Jain, and A. Zakhor. Data processing algorithms for generating textured $3 \mathrm{~d}$ building facade meshes from laser scans and camera images. International Journal of Computer Vision, 2005, 61(2):159-184, February 2005. 2

[25] Chuang Gan, Hang Zhao, Peihao Chen, David Cox, and Antonio Torralba. Self-supervised moving vehicle tracking with stereo sound. In Proceedings of the IEEE International Conference on Computer Vision, pages 7053-7062, 2019. 3

[26] Rohit Girdhar, Du Tran, Lorenzo Torresani, and Deva Ramanan. Distinit: Learning video representations without a single labeled video. In Proceedings of the IEEE International Conference on Computer Vision, pages 852-861, 2019. 3

[27] Aleksey Golovinskiy, Vladimir G Kim, and Thomas Funkhouser. Shape-based recognition of $3 \mathrm{~d}$ point clouds in urban environments. In 2009 IEEE 12th International Conference on Computer Vision, pages 2154-2161. IEEE, 2009. 2

[28] Benjamin Graham, Martin Engelcke, and Laurens van der Maaten. 3d semantic segmentation with submanifold sparse convolutional networks. In Proceedings of the IEEE conference on computer vision and pattern recognition, pages 9224-9232, 2018. 2, 6, 14

[29] Yulan Guo, Hanyun Wang, Qingyong Hu, Hao Liu, Li Liu, and Mohammed Bennamoun. Deep learning for $3 \mathrm{~d}$ point clouds: A survey. IEEE transactions on pattern analysis and machine intelligence, 2020. 2

[30] Saurabh Gupta, Judy Hoffman, and Jitendra Malik. Cross modal distillation for supervision transfer. In Proceedings of the IEEE conference on computer vision and pattern recognition, pages 2827-2836, 2016. 3

[31] Timo Hackel, N. Savinov, L. Ladicky, Jan D. Wegner, K. Schindler, and M. Pollefeys. SEMANTIC3D.NET: A new large-scale point cloud classification benchmark. In ISPRS Annals of the Photogrammetry, Remote Sensing and Spatial Information Sciences, volume IV-1-W1, pages 91-98, 2017. 1

[32] Lei Han, Tian Zheng, Lan Xu, and Lu Fang. Occuseg: Occupancy-aware $3 \mathrm{~d}$ instance segmentation. In Proceedings of the IEEE/CVF Conference on Computer Vision and Pattern Recognition, pages 2940-2949, 2020. 2

[33] Rana Hanocka, Amir Hertz, Noa Fish, Raja Giryes, Shachar Fleishman, and Daniel Cohen-Or. Meshonn: a network with an edge. ACM Transactions on Graphics (TOG), 38(4):1-12, 2019. 2

[34] Carsten Hatger and Claus Brenner. Extraction of road geometry parameters from laser scanning and existing databases. International Archives of Photogrammetry, Remote Sensing and Spatial Information Sciences, 34 (Part 3/W13):225-230, 2003. 2

[35] Elmar Haussmann, Michele Fenzi, Kashyap Chitta, Jan Ivanecky, Hanson Xu, Donna Roy, Akshita Mittel, Nicolas Koumchatzky, Clement Farabet, and Jose M Alvarez. Scalable active learning for object detection. In 2020 IEEE Intelligent Vehicles Symposium (IV), pages 1430-1435. IEEE, 2020. 5

[36] Alexander Hermans, Georgios Floros, and Bastian Leibe. Dense 3d semantic mapping of indoor scenes from rgb-d images. In 2014 IEEE International Conference on Robotics and Automation (ICRA), pages 2631-2638. IEEE, 2014. 2, 6

[37] Hesai. https://scale.com/open-datasets/pandaset, 2020. 1, 2

[38] Zeyu Hu, Mingmin Zhen, Xuyang Bai, Hongbo Fu, and Chiew-lan Tai. Jsenet: Joint semantic segmentation and edge detection network for 3d point clouds. In ECCV, 2020. 2 
[39] Jingwei Huang, Haotian Zhang, Li Yi, Thomas Funkhouser, Matthias Nießner, and Leonidas J Guibas. Texturenet: Consistent local parametrizations for learning from highresolution signals on meshes. In Proceedings of the IEEE Conference on Computer Vision and Pattern Recognition, pages 4440-4449, 2019. 2

[40] Juan Eugenio Iglesias, Ender Konukoglu, Albert Montillo, Zhuowen Tu, and Antonio Criminisi. Combining generative and discriminative models for semantic segmentation of ct scans via active learning. In Biennial International Conference on Information Processing in Medical Imaging, pages 25-36. Springer, 2011. 5

[41] Anttoni Jaakkola, Juha Hyyppa, Hannu Hyyppa, and Antero Kukko. Retrieval algorithms for road surface modelling using laser-based mobile mapping. Sensors, 8(9):5238-5249, 2008. 2

[42] Maximilian Jaritz, Jiayuan Gu, and Hao Su. Multi-view pointnet for $3 \mathrm{~d}$ scene understanding. In Proceedings of the IEEE International Conference on Computer Vision Workshops, pages 0-0, 2019. 2

[43] Maximilian Jaritz, Tuan-Hung Vu, Raoul de Charette, Emilie Wirbel, and Patrick Pérez. xmuda: Cross-modal unsupervised domain adaptation for $3 \mathrm{~d}$ semantic segmentation. In Proceedings of the IEEE/CVF Conference on Computer Vision and Pattern Recognition, pages 12605-12614, 2020. 2

[44] Maximilian Jaritz, Tuan-Hung Vu, Raoul de Charette, Émilie Wirbel, and Patrick Pérez. Cross-modal learning for domain adaptation in $3 \mathrm{~d}$ semantic segmentation. arXiv preprint arXiv:2101.07253, 2021. 2

[45] Longlong Jing, Yucheng Chen, Ling Zhang, Mingyi He, and Yingli Tian. Self-supervised modal and view invariant feature learning. arXiv preprint arXiv:2005.14169, 2020. 3

[46] Tejaswi Kasarla, G Nagendar, Guruprasad M. Hegde, V. Balasubramanian, and C.V. Jawahar. Region-based active learning for efficient labeling in semantic segmentation. In 2019 IEEE Winter Conference on Applications of Computer Vision (WACV), pages 1109-1117, 2019. 5

[47] A Sophia Koepke, Olivia Wiles, Yael Moses, and Andrew Zisserman. Sight to sound: An end-to-end approach for visual piano transcription. In ICASSP 2020-2020 IEEE International Conference on Acoustics, Speech and Signal Processing (ICASSP), pages 1838-1842. IEEE, 2020. 3

[48] Ksenia Konyushkova, Raphael Sznitman, and Pascal Fua. Learning active learning from data. In I. Guyon, U. V. Luxburg, S. Bengio, H. Wallach, R. Fe rgus, S. Vishwanathan, and R. Garnett, editors, Advances in Neural Information Processing Systems, volume 30. Curran Associates, Inc., 2017. 5

[49] Bruno Korbar, Du Tran, and Lorenzo Torresani. Cooperative learning of audio and video models from self-supervised synchronization. In Advances in Neural Information Processing Systems, pages 7763-7774, 2018. 3

[50] Abhijit Kundu, Xiaoqi Yin, Alireza Fathi, David Ross, Brian Brewington, Thomas Funkhouser, and Caroline Pantofaru. Virtual multi-view fusion for $3 \mathrm{~d}$ semantic segmentation. In European Conference on Computer Vision, pages 518-535. Springer, 2020. 2
[51] F. Lafarge, X. Descombes, J. Zerubia, and M. PierrotDeseilligny. Building reconstruction from a single dem. Computer Vision and Pattern Recognition, pages 1-8, June 2008. 2

[52] K. Lai, L. Bo, and D. Fox. Unsupervised feature learning for $3 \mathrm{~d}$ scene labeling. In 2014 IEEE International Conference on Robotics and Automation (ICRA), pages 3050-3057, 2014. 2

[53] John Lambert, Zhuang Liu, Ozan Sener, James Hays, and Vladlen Koltun. Mseg: A composite dataset for multidomain semantic segmentation. In Proceedings of the IEEE/CVF Conference on Computer Vision and Pattern Recognition, pages 2879-2888, 2020. 2, 11

[54] Felix Järemo Lawin, Martin Danelljan, Patrik Tosteberg, Goutam Bhat, Fahad Shahbaz Khan, and Michael Felsberg. Deep projective $3 \mathrm{~d}$ semantic segmentation. In International Conference on Computer Analysis of Images and Patterns, pages 95-107. Springer, 2017. 3

[55] Tsung-Yi Lin, Michael Maire, Serge Belongie, James Hays, Pietro Perona, Deva Ramanan, Piotr Dollár, and C Lawrence Zitnick. Microsoft coco: Common objects in context. In European conference on computer vision, pages 740-755. Springer, 2014. 2

[56] Weiping Liu, Jia Sun, Wanyi Li, Ting Hu, and Peng Wang. Deep learning on point clouds and its application: A survey. Sensors, 19(19):4188, 2019. 2

[57] Lingni Ma, Jörg Stückler, Christian Kerl, and Daniel Cremers. Multi-view deep learning for consistent semantic mapping with rgb-d cameras. In 2017 IEEE/RSJ International Conference on Intelligent Robots and Systems (IROS), pages 598-605. IEEE, 2017. 2

[58] C. Mallet, U. Soergel, and F. Bretar. Analysis of fullwaveform lidar data for an accurate classification of urban areas. In ISPRS08, 2008. 2

[59] Ruben Mascaro, Lucas Teixeira, and Margarita Chli. Diffuser: Multi-view 2d-to-3d label diffusion for semantic scene segmentation. In IEEE International Conference on Robotics and Automation (ICRA 2021)(virtual), 2021. 2

[60] John McCormac, Ankur Handa, Andrew Davison, and Stefan Leutenegger. Semanticfusion: Dense 3d semantic mapping with convolutional neural networks. In 2017 IEEE International Conference on Robotics and automation (ICRA), pages 4628-4635. IEEE, 2017. 2

[61] Johannes Meyer, Andreas Eitel, Thomas Brox, and Wolfram Burgard. Improving unimodal object recognition with multimodal contrastive learning. In IEEE/RSJ International Conference on Intelligent Robots and Systems, 2020. 3

[62] A. Milioto, I. Vizzo, J. Behley, and C. Stachniss. RangeNet++: Fast and Accurate LiDAR Semantic Segmentation. In IEEE/RSJ Intl. Conf. on Intelligent Robots and Systems (IROS), 2019. 2, 10

[63] Arsha Nagrani, Chen Sun, David Ross, Rahul Sukthankar, Cordelia Schmid, and Andrew Zisserman. Speech2action: Cross-modal supervision for action recognition. In Proceedings of the IEEE Conference on Computer Vision and Pattern Recognition, 2020. 3

[64] Gerhard Neuhold, Tobias Ollmann, Samuel Rota Bulo, and Peter Kontschieder. The mapillary vistas dataset for semantic 
understanding of street scenes. In Proceedings of the IEEE International Conference on Computer Vision, pages 49904999, 2017. 2

[65] Mathias Ortner, Xavier Descombes, and Josiane Zerubia. Building outline extraction from digital elevation models using marked point processes. Int. J. Comput. Vision, 72(2):107-132, 2007. 2

[66] Andrew Owens, Jiajun Wu, Josh H. McDermott, William T. Freeman, and Antonio Torralba. Ambient sound provides supervision for visual learning. In Proceedings of the European Conference on Computer Vision (ECCV), 2016. 3

[67] Yancheng Pan, Biao Gao, Jilin Mei, Sibo Geng, Chengkun Li, and Huijing Zhao. Semanticposs: A point cloud dataset with large quantity of dynamic instances, 2020. 1

[68] Hanspeter Pfister, Matthias Zwicker, Jeroen Van Baar, and Markus Gross. Surfels: Surface elements as rendering primitives. In Proceedings of the 27th annual conference on Computer graphics and interactive techniques, pages 335-342, 2000. 4

[69] Quang-Hieu Pham, Thanh Nguyen, Binh-Son Hua, Gemma Roig, and Sai-Kit Yeung. Jsis3d: joint semantic-instance segmentation of $3 \mathrm{~d}$ point clouds with multi-task pointwise networks and multi-value conditional random fields. In Proceedings of the IEEE Conference on Computer Vision and Pattern Recognition, pages 8827-8836, 2019. 2

[70] Charles R Qi, Hao Su, Kaichun Mo, and Leonidas J Guibas. Pointnet: Deep learning on point sets for $3 \mathrm{~d}$ classification and segmentation. In Proceedings of the IEEE conference on computer vision and pattern recognition, pages 652-660, 2017. 2

[71] Charles Ruizhongtai Qi, Li Yi, Hao Su, and Leonidas J Guibas. Pointnet++: Deep hierarchical feature learning on point sets in a metric space. In Advances in neural information processing systems, pages 5099-5108, 2017. 2, 11

[72] Gernot Riegler, Ali Osman Ulusoy, and Andreas Geiger. Octnet: Learning deep 3d representations at high resolutions. In Proceedings of the IEEE Conference on Computer Vision and Pattern Recognition, pages 3577-3586, 2017. 2

[73] Xavier Roynard, Jean-Emmanuel Deschaud, and François Goulette. Paris-lille-3d: A large and high-quality groundtruth urban point cloud dataset for automatic segmentation and classification. The International Journal of Robotics Research, 37(6):545-557, 2018. 1, 2

[74] Olga Russakovsky, Jia Deng, Hao Su, Jonathan Krause, Sanjeev Satheesh, Sean Ma, Zhiheng Huang, Andrej Karpathy, Aditya Khosla, Michael Bernstein, Alexander C. Berg, and Li Fei-Fei. ImageNet Large Scale Visual Recognition Challenge. International Journal of Computer Vision (IJCV), 115(3):211-252, 2015. 15

[75] Burr Settles. Active learning literature survey. CS Technical Reports, 2009. 5

[76] Shaoshuai Shi, Chaoxu Guo, Li Jiang, Zhe Wang, Jianping Shi, Xiaogang Wang, and Hongsheng Li. PV-RCNN: Pointvoxel feature set abstraction for $3 \mathrm{~d}$ object detection. In Proceedings of the IEEE/CVF Conference on Computer Vision and Pattern Recognition, pages 10529-10538, 2020. 2

[77] Yawar Siddiqui, Julien Valentin, and Matthias Niessner. Viewal: Active learning with viewpoint entropy for semantic segmentation. In Proceedings of the IEEE/CVF Conference on Computer Vision and Pattern Recognition (CVPR), June 2020. 5

[78] Shuran Song, Fisher Yu, Andy Zeng, Angel X Chang, Manolis Savva, and Thomas Funkhouser. Semantic scene completion from a single depth image. In Proceedings of the IEEE Conference on Computer Vision and Pattern Recognition, pages 1746-1754, 2017. 2

[79] Haotian Tang, Zhijian Liu, Shengyu Zhao, Yujun Lin, Ji Lin, Hanrui Wang, and Song Han. Searching efficient 3d architectures with sparse point-voxel convolution. In European Conference on Computer Vision, 2020. 9

[80] Hugues Thomas, Charles R Qi, Jean-Emmanuel Deschaud, Beatriz Marcotegui, François Goulette, and Leonidas J Guibas. Kpconv: Flexible and deformable convolution for point clouds. In Proceedings of the IEEE International Conference on Computer Vision, pages 6411-6420, 2019. 2

[81] Yonglong Tian, Dilip Krishnan, and Phillip Isola. Contrastive multiview coding. In Computer Vision-ECCV 2020: 16th European Conference, Glasgow, UK, August 23-28, 2020, Proceedings, Part XI 16, pages 776-794. Springer, 2020. 3

[82] M. Torre and P.I. Radeva. Agricultural-field extraction on aerial images by region competition algorithm. In ICPR, pages Vol I: 313-316, 2000. 2

[83] Alexander Vezhnevets, Joachim M Buhmann, and Vittorio Ferrari. Active learning for semantic segmentation with expected change. In 2012 IEEE conference on computer vision and pattern recognition, pages 3162-3169. IEEE, 2012. 5

[84] Vibhav Vineet, Ondrej Miksik, Morten Lidegaard, Matthias Nießner, Stuart Golodetz, Victor A Prisacariu, Olaf Kähler, David W Murray, Shahram Izadi, Patrick Pérez, et al. Incremental dense semantic stereo fusion for large-scale semantic scene reconstruction. In 2015 IEEE International Conference on Robotics and Automation (ICRA), pages 75-82. IEEE, 2015. 2

[85] Sourabh Vora, Alex H Lang, Bassam Helou, and Oscar Beijbom. Pointpainting: Sequential fusion for $3 \mathrm{~d}$ object detection. In Proceedings of the IEEE/CVF Conference on Computer Vision and Pattern Recognition, pages 4604-4612, 2020. 2

[86] Haiyan Wang, Xuejian Rong, Liang Yang, Shuihua Wang, and Yingli Tian. Towards weakly supervised semantic segmentation in 3D graph-structured point clouds of wild scenes. In $B M V C$, page 284, 2019. 2

[87] Yunsheng Wang, Holger Weinacker, and Barbara Koch. A lidar point cloud based procedure for vertical canopy structure analysis and $3 \mathrm{~d}$ single tree modelling in forest. Sensors, 8:1424-8220, 2008. 2

[88] Denis F. Wolf, Gaurav S. Sukhatme, Dieter Fox, and Wolfram Burgard. Autonomous terrain mapping and classification using hidden markov models. In IEEE International Conference on Robotics and Automation, pages 2038-2043, April 2005. 2

[89] Shuai Xie, Zunlei Feng, Ying Chen, Songtao Sun, Chao Ma, and Mingli Song. Deal: Difficulty-aware active learning for semantic segmentation. In Proceedings of the Asian Conference on Computer Vision (ACCV), November 2020. 5 
[90] Chenfeng Xu, Bichen Wu, Zining Wang, Wei Zhan, Peter Vajda, Kurt Keutzer, and Masayoshi Tomizuka. Squeezesegv3: Spatially-adaptive convolution for efficient pointcloud segmentation. In European Conference on Computer Vision, pages 1-19. Springer, 2020. 2

[91] Hui Xu, Nathan Gossett, and Baoquan Chen. Knowledge and heuristic-based modeling of laser-scanned trees. ACM Trans. Graph., 26(4):19, 2007. 2

[92] Li Yi, Boqing Gong, and Thomas Funkhouser. Complete \& label: A domain adaptation approach to semantic segmentation of lidar point clouds. In Proceedings of the IEEE/CVF Conference on Computer Vision and Pattern Recognition, pages 15363-15373, 2021. 2

[93] Sijie Yu, Sreenivas R. Sukumar, Andreas F. Koschan, and Mongi A. Abidi. 3d reconstruction of road surfaces using an integrated multi-sensory. Optics and Lasers in Engineering, 45 (7):808-818, February 2007. 2

[94] Cheng Zhang, Zhi Liu, Guangwen Liu, and Dandan Huang. Large-scale $3 \mathrm{~d}$ semantic mapping using monocular vision. In 2019 IEEE 4th International Conference on Image, Vision and Computing (ICIVC), pages 71-76. IEEE, 2019. 2

[95] Yang Zhang, Zixiang Zhou, Philip David, Xiangyu Yue, Zerong Xi, Boqing Gong, and Hassan Foroosh. Polarnet: An improved grid representation for online lidar point clouds semantic segmentation. In Proceedings of the IEEE/CVF Conference on Computer Vision and Pattern Recognition, pages 9601-9610, 2020. 2, 10

[96] Hang Zhao, Chuang Gan, Andrew Rouditchenko, Carl Vondrick, Josh McDermott, and Antonio Torralba. The sound of pixels. In Proceedings of the European Conference on Computer Vision (ECCV), page 570-586, 2018. 3

[97] Jiaping Zhao and Suya You. Road network extraction from airborne lidar data using scene context. In International Workshop on Point Cloud Processing (Affiliated with CVPR 2012), 2012. 2

[98] Xinge Zhu, Hui Zhou, Tai Wang, Fangzhou Hong, Yuexin Ma, Wei Li, Hongsheng Li, and Dahua Lin. Cylindrical and asymmetrical $3 \mathrm{~d}$ convolution networks for lidar segmentation. In Proceedings of the IEEE/CVF Conference on Computer Vision and Pattern Recognition, pages 99399948, 2021. 9, 10 